\title{
Evaluating Reliability Compliance of the Power Utility Distribution Networks
}

\author{
Lambe Mutalub Adesina ${ }^{1 *}$, Kamaldeen Sa'adu' ${ }^{2}$ Ademola Abdulkareem ${ }^{3}$ \\ ${ }^{I}$ Department of Electrical and Computer Engineering, Kwara State University, P.M.B. 234, Malete, Ilorin, Nigeria. \\ ${ }^{2}$ Network Planning Dept., Technical Service Function, Eko Electricity Company, 24/25 Marina Street, Lagos, Nigeria. \\ ${ }^{3}$ Dept. of Electrical and Information Engineering, Covenant University, Sango Ota, Nigeria.
}

ORCID: 0000-0002-3744-046X (Lambe)

\begin{abstract}
Distribution network planners carry out analyses of the power distribution network that may include studies like faults, sensitivity analysis and reliability assessment to enhance the robustness of the network. System reliability is the ability of the power system to provide an adequate supply of electrical power at a desired time without interruption. This paper focuses on reliability assessment of distribution network. It involves the determination of the reliability indices which explain the dependability of the system. Fifteen $11 \mathrm{kV}$ feeders are considered and the required data of the feeders to be used in this study were sourced from the chosen power utility company in Nigeria. A power system software called ETAP was used in computational process. The reliability indices of the chosen network were computed, and the results obtained are presented. From the results, the performance of these Fifteen $11 \mathrm{kV}$ feeders are classified into three; Best, averagely and poorly performing feeders, and they are highlighted using tables and charts. The results are discussed and analyzed. The findings from the study indicate that some feeders have very good reliability indices while some are having average values and others are with poor reliability indices. The most critical feeders that need improvement were identified.
\end{abstract}

Keywords: Feeders, Network reliability, Reliability assessment, Reliability indices, Supply availability

\section{INTRODUCTION}

Electricity plays a vital role in the socio-economic development of any nation, as it constitutes about 35 percent of the total cost of production of goods [1]. Lack of reliable electric supply would therefore reduce the interest of investors due to unprecedented costs of production thus increasing the rate of unemployment as well as extending the poverty range of such a country. Electric power distribution, being the final stage in the delivery of electric power; about $80 \%$ of power system interruptions results from power distribution system failure [2 -12]. Therefore, assessing the reliability of power distribution networks is of great interest.

The reliability of a power distribution system involves its ability to ensure uninterrupted power supply to all its customers in the amount desired, for the period intended and under the operating conditions intended whilst conforming to accepted standards [3, 4, 13 - 15]. Power system reliability can be evaluated using two varieties of techniques - analytical and simulation. The analytical techniques represent the system by a simplified mathematical model and evaluation of the reliability indices from this model using direct mathematical solutions; while simulation techniques, estimate the reliability indices by simulating the actual process and random behaviour of the system $[5,6,16$ - 18]

Furthermore, evaluation of reliability indices is classified into two main categories: repairable and non-repairable [19]. Repairable systems repair and put the system components back into operation after components failure, whereas a nonrepairable system fails to repair system components after components' failure, and thus a new one needs to be replaced $[18,19]$. However, most of the electric power systems' failures are repairable systems [19]. Therefore, effective reliability analysis is an essential factor in operational planning of electric power system $[19,20]$.

Accurate analysis of power system reliability assists in predicting future failure behavior of power system and in making appropriate maintenance plans [19]. Distribution power system reliability is greatly affected by outages caused by various environmental factors on overhead lines. Thus, it is necessary to find out the effects of these outages on power systems operation since animals cause most significant of the outages on overhead distribution systems [21].

This paper is aimed at evaluating the reliability parameters or indices of the selected distribution network of a power utility company in Nigeria. It employed the method of analytic technique. It involves the use of relevant reliability indices generated by ETAP Software using the data obtained from the power utility company. The reliability study of the $11 \mathrm{kV}$ feeders of the selected power utility company in Nigeria were carried out. The results obtained for each of the $11 \mathrm{kV}$ feeders were compared using Excel application.

Several studies have been carried out regarding the reliability of electrical power systems. These studies are besides the nonpower system reliability studies that have been undertaken over the years. However, while some of these power system studies are from a regulatory perspective, others stem from an operational point of view. The following are the related works as regards to power system reliability studies carried out in the past. 
International Journal of Engineering Research and Technology. ISSN 0974-3154, Volume 13, Number 8 (2020), pp. 1940-1956

(C) International Research Publication House. https://dx.doi.org/10.37624/IJERT/13.8.2020.1940-1956

Popoola et al. (2011) presented a paper on the reliability worth of electric power supply in Nigeria. Their report showed how unreliable electricity was in the country. By conducting a survey of customers, they discovered that electric power availability in the country was better during the rainy season than the dry season. Onime and Adegboyega (2014) performed a reliability analysis of Ekpoma network in Edo state, Nigeria. The results from their study showed that there was a networkwide reliability problem with the feeders due to continuous interruptions and voltage fluctuations. Eminoglu and Uyan (2016) used a combination of simulation (Monte Carlo method) and analysis to compute reliability indices using real world information collected from a utility company. They based their study on the Nigide Region's power system. Findings from the study showed that the results from both the analysis and simulation were almost congruous. Aljohani and Beshir (2017) conducted a work on the impact of smart grid applications in increasing distribution network reliability. Among other objectives, their focus was on analysing an IEEE 34 node test feeder to know the best position for installing automatic switching instruments and measure their correct placement using the

Distribution system performance. Akintola (2017) did an analysis of an injection substation in Aguda, Lagos State, Nigeria, where he used information from a power utility to determine the reliability of the substation. The findings from his work indicated that transformer and fuse failures were the major contributors to the unavailability of the substation. Babu (2017) carried out a research and published a dissertation on reliability evaluation of distribution systems, in which he presented some advanced methods for measuring and assessing various failure modes in power distribution systems. The work was conducted in two parts, with the first part concentrated on the effects of failure-related events, and the second part concentrated on optimizing the network while putting cost into consideration. He developed a simulation model for analysing false tripping probabilities. Abunima et al (2018) carried out a review of reliability studies on composite power systems. Their research focused on previously less covered topics relating to power system reliability with the objective of enhancing knowledge of composite power systems reliability. Utilizing articles published in the last twelve years (2007 to 2019), their study, which was mainly investigative and compared the reliability of various composite systems.

With all the itemised work done and the plethora of published literature on electric power system reliability, none of the studies neither use Nigerian power utility networks as case study or apply the results to amend problems associated with reliability of Nigerian networks, particularly the distribution sub-sector of the industry. Most of the available work on reliability assessment do not directly reflect the Nigerian situation, and in the few cases where they do, most of the data used are not real-world data as they are majorly theoretical. Hence, the motivation for this reliability assessment is premised on the insufficiency of related studies on the subject matter as it relates to Nigeria. The results of this study will not only aid the utility company in improving its network reliability but will help to fill a part of this knowledge gap.

\section{RELIABILITY ANALYSIS OF POWER DISTRIBUTION NETWORKS}

The list of symbols and their interpretations as used in this paper is shown in Table 1.

Table 1: Symbols and interpretations

\begin{tabular}{|c|c|}
\hline Symbol & Interpretation \\
\hline MTTF & Mean time to Failure \\
\hline MTTR & Mean time to Repair \\
\hline$M T B F$ & Mean time between Failures \\
\hline SAIDI & System Average Interruption Duration Index \\
\hline SAIFI & System Average Interruption Frequency Index \\
\hline CAIDI & Customer Average Interruption Duration Index \\
\hline$T N C I$ & Total Number of Customer Interruptions \\
\hline TNCS & Total Number of Customers Served \\
\hline$S U M C I D$ & Sum of Customer Interruption Durations \\
\hline$\lambda$ & Failure rate \\
\hline$\mu$ & Repair rate \\
\hline$A$ & Availability \\
\hline $\mathrm{OH}$ & Outage hour \\
\hline$L$ & Load shedding period \\
\hline$F$ & Fault \\
\hline$O N$ & $\begin{array}{l}\text { Feeder on Lowest reading value of Calibration } \\
\text { (Negligible meter reading) }\end{array}$ \\
\hline$D N P$ & $\begin{array}{l}\text { Feeder on Disconnection for Non-Payment of } \\
\text { Monthly Bill }\end{array}$ \\
\hline$H$ & Hour \\
\hline$F N$ & Feeder Name \\
\hline$N F$ & Number of Faults \\
\hline$N C C$ & $\begin{array}{l}\text { Number of Customers Served (Connected } \\
\text { Customers) }\end{array}$ \\
\hline
\end{tabular}




\section{II.I Mathematical Equations of Reliability}

The data required for reliability studies can be obtained from the data sheet of the selected power utility company. Among these data are, outage hours $(\mathrm{OH})$, service hours $(\mathrm{SH})$, Number of Faults (NF), Force Outage Hours (FOH) etc. Thus, the performance of the network reliability is also obtainable using the following mathematical relations [1, 3, 5-15];

Outage Hour $(\mathrm{OH})=\mathrm{FOH}+\mathrm{LH}+\mathrm{POH}$.

Where,

$\mathrm{FOH}=$ Forced Outage hours (Total duration of fault)

LH $=$ Load-shedding hours

$\mathrm{POH}=$ Planned outage hours

Hours $(\mathrm{H})=$ Number of days $\times 24$ hours

Service Hours $(\mathrm{SH})=\mathrm{H}-\mathrm{OH}$

Mean Time to Failure $(\mathrm{MTTF})=\frac{S H}{N F}$

Mean Time to Repair (MTTR) $=\frac{F O H}{N F}$.

Mean Time between Failure $(\mathrm{MTBF})=\mathrm{MTTF}+\mathrm{MTTR}$.

Failure rate $(\lambda)=\frac{1}{M T T F}$

Repair rate $(\mu)=\frac{1}{M T T R}$.

Inherent Availability (IA) $=\frac{M T B F}{M T B F+M T T R}$.

SAIFI $=\frac{\text { Total Number of Customer Interruptions }}{\text { Total Number of Customers Served }}$

SAIDI $=\frac{\text { CCustomer Interruption Durations }}{\text { Total Number of Customers Served }}$.

CAIDI $=\frac{\sum \text { Customer Interruption Durations }}{\text { Total Number of Customer Interruptions }}$.

\section{II.II Developed Algorithm}

The developed algorithm has the following listed procedures:

1. Start

2. Obtain the $11 \mathrm{kV}$ feeders' data from the selected Power utility company.

3. Input annual data for all the $11 \mathrm{kV}$ feeders. These data include, Number of Faults (NF), Forced Outage Hours (FOH), Load Shedding Hours (H), Planned Outage Hours (POH), Service Hours (SH), Total Number of customers served (TNCS), Total Number of Customer Interrupted (TNCI), Sum of Customer Interruption Durations (SUMCID)

4. Set Counter $K=1$

5. Compute the reliability indices that include; Outage Hour $\mathrm{OH}(\mathrm{K})$, Operational Availability $\mathrm{OA}(\mathrm{K}), \operatorname{MTTF}(\mathrm{K})$, $\operatorname{MTTR}(\mathrm{K}), \operatorname{MTBF}(\mathrm{K})$, Failure rate $\lambda(\mathrm{K})$ and Repair rate $\mu(\mathrm{k})$ using equations (1), (3), ( 4), ( 5), (6), (7) and (8) respectively and SAIFI (K), SAIDI (K), CAIDI (K) and $\mathrm{A}(\mathrm{K})$ using equations (10), (11), (12) and (9) respectively

6. Is $\mathrm{K}=15$, If No GOTO $4: \mathrm{K}=\mathrm{K}+1$

7. Print OA (K), MTTF (K), MTTR (K), MTBF (K), $\lambda(K)$, $\mu(\mathrm{k})$.

8. Print SAIFI (K), SAIDI (K), CAIDI (K), IA(K)

9. End

\section{II.III Network Case Study and Data Collection}

An $11 \mathrm{kV}$ network of a leading power utility in Nigeria is selected and used as case study. It comprises of fifteen $11 \mathrm{kV}$ feeders that are interconnected. A 12-hour data of the bi-hourly readings (in megawatts) taken from each of the 15 feeders in the coverage area were obtained from the selected power distribution company as shown in Table 2 [22, 23]. Obtained data shows that outages data were given as a bulk i.e not separated into various classes of outages. In such situation, $30 \%$ of overall outages is reasonably taken as forced outage hours $(\mathrm{FOH})$ in order to estimate the MTTR values of each feeder. With all the data available, an ETAP, a software package was employed in assessing the reliability of the feeders considered in the study. The Flowchart in Fig. 1 below pictorially illustrates the details of the algorithm described in subsection II.II above. The results are presented in section 4.0.

\section{THE RESULTS OF RELIABILITY STUDY}

Electricity distribution operational record book of the case study network was picked up and parameters such as those contained in Table 3 were extracted. An ETAP computer software package was applied to the algorithm described in subsection II.I above. Equations (3), (4), (5), (6), (7) and (8) yield the results in Table 4 . 


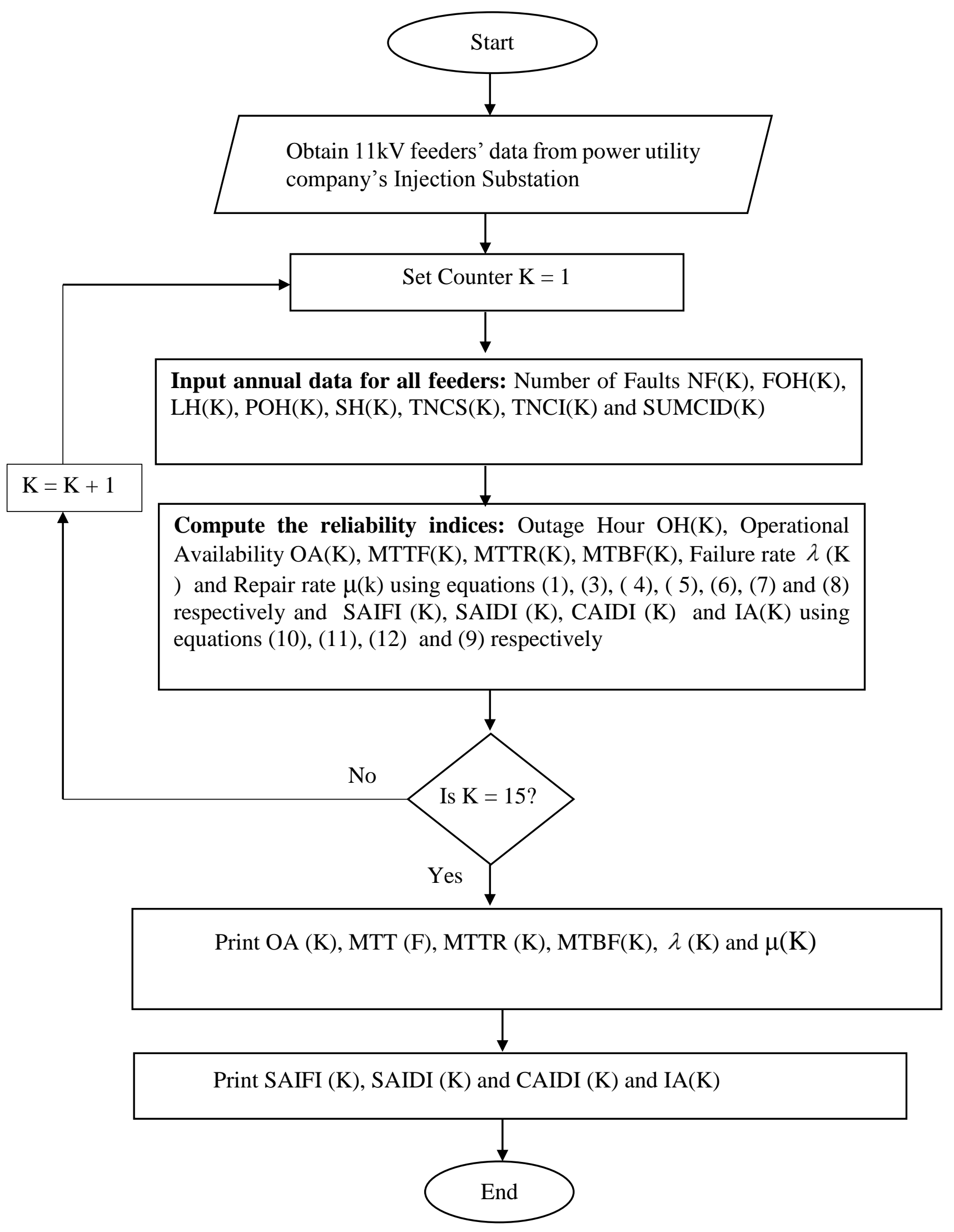

Fig. 1: Flowchart showing the details of the developed algorithm. 
International Journal of Engineering Research and Technology. ISSN 0974-3154, Volume 13, Number 8 (2020), pp. 1940-1956 (C) International Research Publication House. https://dx.doi.org/10.37624/IJERT/13.8.2020.1940-1956

Table 2: Recorded 2-hourly readings (Megawatts) for the 15 No. $11 \mathrm{kV}$ feeders at Power Utility Control Room.

\begin{tabular}{|c|c|c|c|c|c|c|c|c|c|c|c|c|c|}
\hline FN & $\begin{array}{l}0200 \\
\text { h }\end{array}$ & $\begin{array}{l}0400 \\
\text { h }\end{array}$ & $\begin{array}{l}0600 \\
\text { h }\end{array}$ & $\begin{array}{l}0800 \\
\text { h }\end{array}$ & $\begin{array}{l}0100 \\
0 h\end{array}$ & $\begin{array}{l}1200 \\
\text { h }\end{array}$ & $\begin{array}{l}1400 \\
\text { h }\end{array}$ & $\begin{array}{l}1600 \\
\text { h }\end{array}$ & $\begin{array}{l}1800 \\
\text { h }\end{array}$ & $\begin{array}{l}2000 \\
\text { h }\end{array}$ & $\begin{array}{l}2200 \\
h\end{array}$ & $\begin{array}{l}2400 \\
\text { h }\end{array}$ & TNCS \\
\hline Al & 1.6 & 1.5 & 1.5 & 1.5 & 1.6 & 1.7 & 1.6 & 1.6 & 0.7 & 1.7 & 1.8 & 1.8 & 68 \\
\hline A2 & 0.2 & 0.2 & 0.2 & 0.2 & 0.2 & 0.2 & 0.2 & 0.2 & 0.2 & 0.2 & 0.2 & 0.2 & 56 \\
\hline $\mathbf{A 3}$ & 1 & 1 & 1 & 0.9 & 0.9 & 0.9 & 0.9 & 0.9 & 0.9 & 1 & 1 & 1 & 88 \\
\hline A4 & 1.3 & 1.3 & 1.3 & 1.3 & 1.3 & 1.3 & 1.2 & 1.2 & 1.2 & 1.2 & 1.3 & 1.3 & 45 \\
\hline A5 & 1.5 & 1.5 & 1.6 & 1.6 & 1.5 & 1.5 & 1.2 & 1.4 & 1.5 & 1.4 & 1.4 & 1.5 & 66 \\
\hline A16 & 0.9 & 0.9 & 0.8 & 0.8 & 0.8 & 0.8 & 0.8 & 0.8 & 0.7 & 0.7 & 0.7 & 0.7 & 65 \\
\hline A37 & 2.6 & 2.6 & 2.6 & 2.6 & 2.6 & 2.6 & L & $\mathrm{L}$ & L & L & $\mathrm{L}$ & L & 56 \\
\hline A46 & 0.5 & 0.5 & 0.5 & 0.5 & 0.5 & 0.5 & 0.4 & 0.4 & 0.5 & 0.5 & 0.5 & 0.5 & 89 \\
\hline A47 & 0.6 & 0.6 & 0.6 & 0.6 & 0.6 & 0.7 & 0.6 & 0.6 & 0.6 & 0.6 & 0.6 & 0.6 & 99 \\
\hline A48 & 1.4 & 1.4 & 1.4 & 1.4 & 1.4 & 1.4 & 1.4 & 1.4 & 1.5 & 1.5 & 1.5 & 1.5 & 168 \\
\hline A49 & 0.1 & 0.1 & 0.1 & 0.1 & 0.1 & 0.1 & 0.1 & 0.1 & 0.1 & 0.1 & 0.1 & 0.1 & 85 \\
\hline A50 & 2.1 & 2 & 2 & 1.9 & 1.5 & 1 & 1 & 1 & 1 & 1 & 1 & 1 & 97 \\
\hline A57 & ON & $\mathrm{ON}$ & $\mathrm{ON}$ & $\mathrm{ON}$ & $\mathrm{ON}$ & $\mathrm{ON}$ & $\mathrm{ON}$ & ON & $\mathrm{ON}$ & $\mathrm{ON}$ & $\mathrm{ON}$ & $\mathrm{ON}$ & 0 \\
\hline A70 & 0.1 & $\mathrm{ON}$ & ON & ON & $\mathrm{ON}$ & $\mathrm{ON}$ & $\mathrm{ON}$ & $\mathrm{ON}$ & $\mathrm{ON}$ & $\mathrm{ON}$ & 0.1 & 0.1 & 0 \\
\hline A71 & 1.3 & 1.2 & 1.2 & 1.2 & 1.2 & 1.2 & 1.2 & 1.3 & 1.3 & 1.3 & 1.3 & 1.3 & 77 \\
\hline
\end{tabular}

Table 3: Annual recorded faults, Service hours, and Outage hours for 15 no. $11 \mathrm{kV}$ feeders.

\begin{tabular}{|c|c|c|c|c|c|}
\hline $\begin{array}{l}\text { Feeder } \\
\text { Name }\end{array}$ & $\begin{array}{l}\text { No. of Faults } \\
\text { Per Annum }\end{array}$ & $\begin{array}{c}\text { Service Hours } \\
\text { Per Annum (SH) }\end{array}$ & $\begin{array}{l}\text { Outage Hours } \\
\text { Per Annum } \\
(\mathrm{OH})\end{array}$ & $\begin{array}{c}\text { Customers } \\
\text { affected with } \\
\text { Faults Annually }\end{array}$ & TNCS \\
\hline A1 & 1 & 8491.890909 & 268.11 & 6 & 68 \\
\hline A2 & 2 & 8312.709091 & 447.29 & 15 & 56 \\
\hline A3 & 1 & 8246.345455 & 513.65 & 25 & 88 \\
\hline A4 & 11 & 8213.16 & 546.84 & 30 & 45 \\
\hline A5 & 4 & 8125.56 & 634.44 & 26 & 66 \\
\hline A16 & 26 & 7116.84 & 1643.16 & 65 & 65 \\
\hline A37 & 5 & 7112.85 & 1647.15 & 30 & 56 \\
\hline A46 & 35 & 6389.49 & 2370.51 & 89 & 89 \\
\hline A47 & 32 & 6382.86 & 2377.15 & 99 & 99 \\
\hline A48 & 90 & 6374.89 & 2385.11 & 168 & 168 \\
\hline A49 & 15 & 6319.15 & 2440.86 & 60 & 85 \\
\hline A50 & 13 & 6304.55 & 2455.46 & 40 & 97 \\
\hline A57 & 36 & 7114.18 & 1645.82 & 0 & 0 \\
\hline A70 & 7 & 7220.36 & 1539.00 & 0 & 0 \\
\hline A71 & 41 & 7209.75 & 1550.25 & 77 & 77 \\
\hline
\end{tabular}


International Journal of Engineering Research and Technology. ISSN 0974-3154, Volume 13, Number 8 (2020), pp. 1940-1956 (C) International Research Publication House. https://dx.doi.org/10.37624/IJERT/13.8.2020.1940-1956

Table 4: Estimated annual supply operational availability, Failure rate, Repair rate.

\begin{tabular}{|c|c|c|c|c|c|c|}
\hline $\begin{array}{l}\text { Feeder } \\
\text { Name }\end{array}$ & $\begin{array}{l}\text { Annual Operational } \\
\text { Availability (SH) }\end{array}$ & $\begin{array}{l}\text { MTTF } \\
\text { (Hours) }\end{array}$ & $\begin{array}{l}\text { MTTR } \\
\text { (Hours) }\end{array}$ & MTBF & $\begin{array}{l}\text { System } \\
\text { Failure } \\
\text { Rate }(\lambda)\end{array}$ & $\begin{array}{c}\text { System Repair } \\
\text { Rate }(\mu)\end{array}$ \\
\hline A1 & 0.9693 & 8491.89 & 6.6363 & 8498.52 & 0.00012 & 0.151 \\
\hline A2 & 0.9489 & 4156.35 & 22.5636 & 4178.92 & 0.00024 & 0.044 \\
\hline A3 & 0.9413 & 8246.34 & 13.2727 & 8259.62 & 0.00012 & 0.075 \\
\hline A4 & 0.9375 & 746.65 & 14.91 & 761.56 & 0.00134 & 0.07 \\
\hline A5 & 0.9275 & 2031.39 & 47.58 & 2078.97 & 0.00049 & 0.02 \\
\hline A16 & 0.8124 & 273.72 & 18.96 & 292.68 & 0.00365 & 0.05 \\
\hline A37 & 0.8120 & 1422.57 & 98.33 & 1521.40 & 0.00070 & 0.01 \\
\hline A46 & 0.7294 & 182.56 & 20.32 & 202.88 & 0.00548 & 0.05 \\
\hline A47 & 0.7286 & 199.46 & 22.29 & 221.75 & 0.00501 & 0.04 \\
\hline A48 & 0.7277 & 70.83 & 7.75 & 78.78 & 0.01 & 0.13 \\
\hline A49 & 0.7214 & 421.28 & 48.81 & 470.09 & 0.00237 & 0.02 \\
\hline A50 & 0.7197 & 484.97 & 49.11 & 534.08 & 0.00206 & 0.02 \\
\hline A57 & 0.8121 & 197.62 & 13.72 & 211.54 & 0.00506 & 0.07 \\
\hline A70 & 0.8242 & 1031.48 & 65.96 & 1097.44 & 0.00097 & 0.02 \\
\hline A71 & 0.8230 & 175.85 & 11.34 & 187.19 & 0.00569 & 0.09 \\
\hline
\end{tabular}

Table 5: Other annual reliability indices for the $11 \mathrm{kV}$ feeders

\begin{tabular}{|c|c|c|c|c|}
\hline Feeder Name & SAIFI (int) & SAIDI (hr) & CAIDI (hr) & $\begin{array}{c}\text { INHERENT } \\
\text { AVAILABILITY (IA) }\end{array}$ \\
\hline A1 & 0.014706 & 3.942781 & 268.1091 & 1.00 \\
\hline A2 & 0.035714 & 7.987338 & 223.6455 & 0.99 \\
\hline A3 & 0.011364 & 5.836983 & 513.6545 & 0.99 \\
\hline A4 & 0.244444 & 12.15192 & 49.7124 & 0.98 \\
\hline A5 & 0.060606 & 9.612672 & 158.6091 & 0.98 \\
\hline A16 & 0.4 & 25.27944 & 63.1986 & 0.94 \\
\hline A37 & 0.089286 & 29.41331 & 329.4291 & 0.94 \\
\hline A46 & 0.393258 & 26.63493 & 67.72883 & 0.99 \\
\hline A47 & 0.323232 & 24.01157 & 74.2858 & 0.91 \\
\hline A48 & 0.535714 & 14.19708 & 26.50121 & 0.91 \\
\hline A49 & 0.176471 & 28.71594 & 162.7236 & 0.91 \\
\hline A50 & 0.134021 & 25.31396 & 188.8811 & 0.92 \\
\hline A57 & 0 & 0 & 0 & 0.94 \\
\hline A70 & 0 & 0 & 0 & 0.94 \\
\hline A71 & 0.532468 & 20.13318 & 37.81109 & 0.94 \\
\hline
\end{tabular}


International Journal of Engineering Research and Technology. ISSN 0974-3154, Volume 13, Number 8 (2020), pp. 1940-1956 (C) International Research Publication House. https://dx.doi.org/10.37624/IJERT/13.8.2020.1940-1956

Table 6: Operational availability for the best performing feeders

\begin{tabular}{|c|c|c|c|c|}
\hline $\begin{array}{c}\text { FEEDER } \\
\text { NAME }\end{array}$ & $\begin{array}{c}\text { ANNUAL NO } \\
\text { OF FAULTS }\end{array}$ & $\begin{array}{c}\text { TOTAL ANNUAL } \\
\text { SERVICE HOURS }\end{array}$ & $\begin{array}{c}\text { TOTAL ANNUAL } \\
\text { OUTAGE HOURS }\end{array}$ & $\begin{array}{c}\text { OPERATIONAL } \\
\text { AVAILABILITY }\end{array}$ \\
\hline $\mathrm{A} 1$ & 1 & 8491.890909 & 268.1090909 & 0.969393939 \\
\hline $\mathrm{A} 2$ & 2 & 8312.709091 & 447.2909091 & 0.948939394 \\
\hline $\mathrm{A} 3$ & 1 & 8246.345455 & 513.6545455 & 0.941363636 \\
\hline $\mathrm{A} 4$ & 11 & 8213.163636 & 546.8363636 & 0.937575758 \\
\hline $\mathrm{A} 5$ & 4 & 8125.563636 & 634.4363636 & 0.927575758 \\
\hline
\end{tabular}

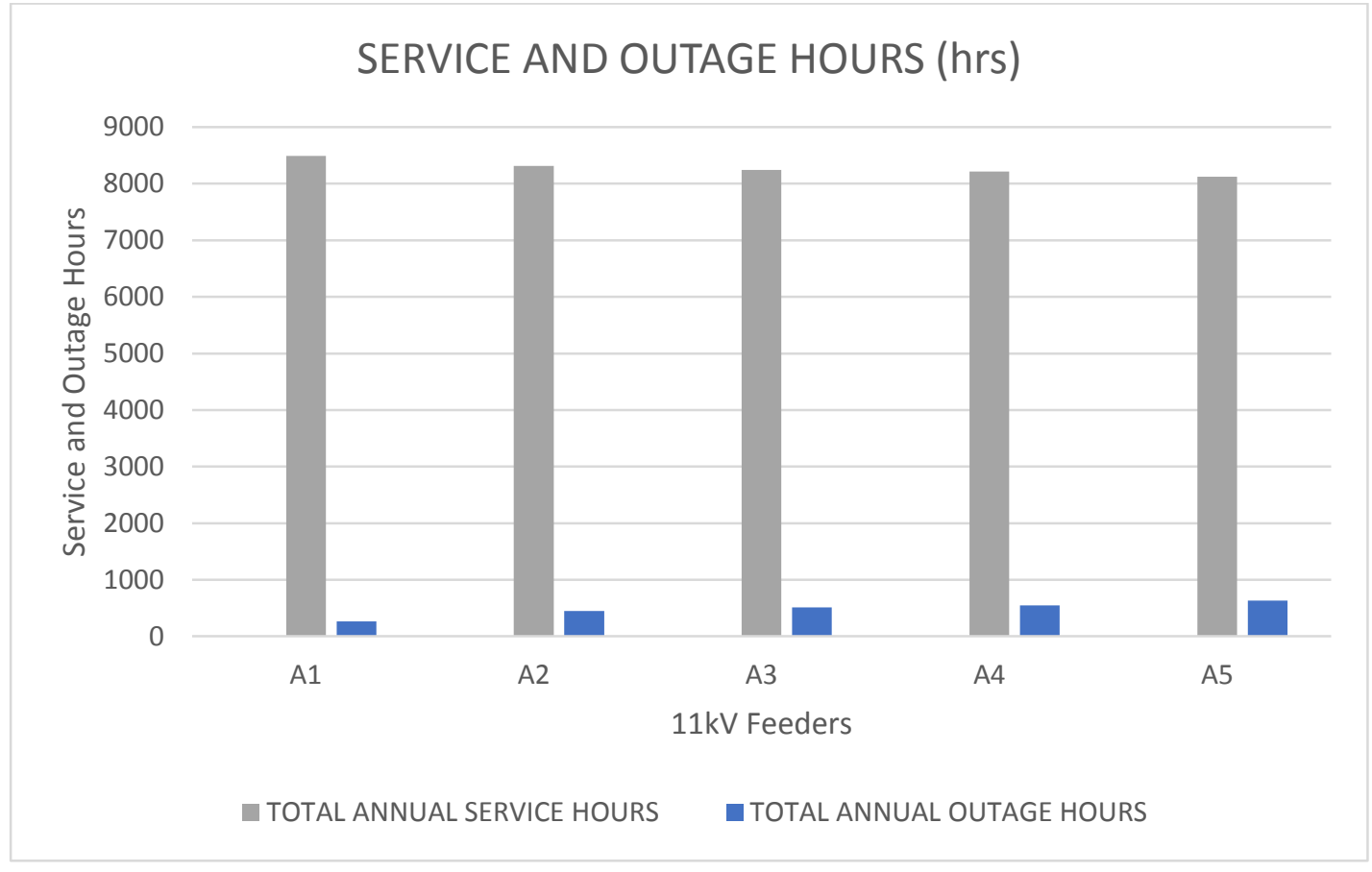

Fig. 2a: Service and Outage hours for the best performing feeders

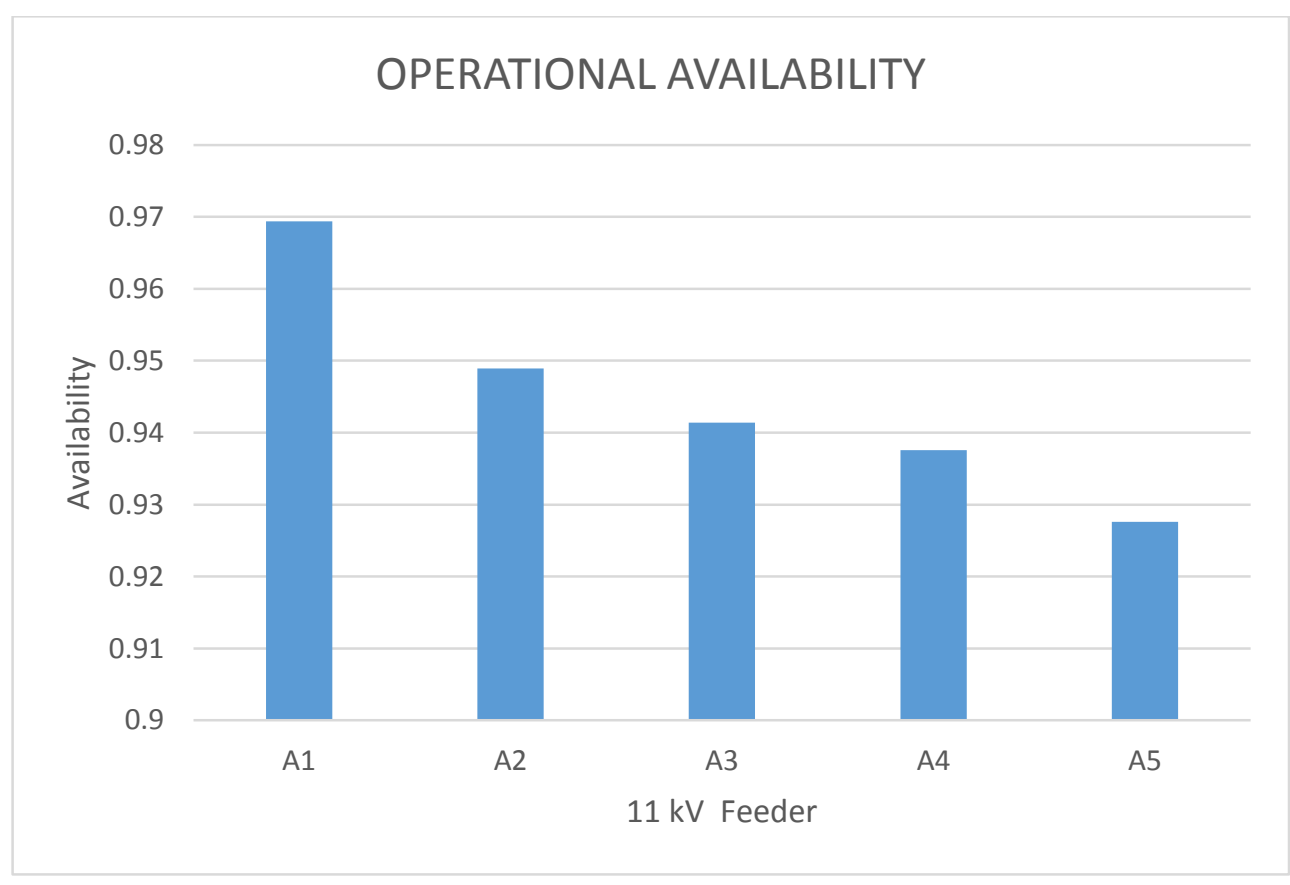

Fig. 2b: Operational availability for the best performing feeders 
International Journal of Engineering Research and Technology. ISSN 0974-3154, Volume 13, Number 8 (2020), pp. 1940-1956 (C) International Research Publication House. https://dx.doi.org/10.37624/IJERT/13.8.2020.1940-1956

Table 7: Operational availability for the averagely performing feeders

\begin{tabular}{|c|c|c|c|c|}
\hline $\begin{array}{c}\text { FEEDER } \\
\text { NAME }\end{array}$ & $\begin{array}{c}\text { ANNUAL NO } \\
\text { OF FAULTS }\end{array}$ & $\begin{array}{c}\text { TOTAL ANNUAL } \\
\text { SERVICE HOURS }\end{array}$ & $\begin{array}{c}\text { TOTAL ANNUAL } \\
\text { OUTAGE HOURS }\end{array}$ & $\begin{array}{c}\text { OPERATIONAL } \\
\text { AVAILABILITY }\end{array}$ \\
\hline A16 & 26 & 7116.836364 & 1643.163636 & 0.812424242 \\
\hline A37 & 5 & 7112.854545 & 1647.145455 & 0.811969676 \\
\hline A57 & 36 & 7114.181818 & 1645.818182 & 0.812121212 \\
\hline A70 & 7 & 7220.363636 & 1539.636364 & 0.824242424 \\
\hline A71 & 41 & 7209.745455 & 1550.254545 & 0.823030303 \\
\hline
\end{tabular}

\section{SERVICE AND OUTAGE HOURS(hrs)}

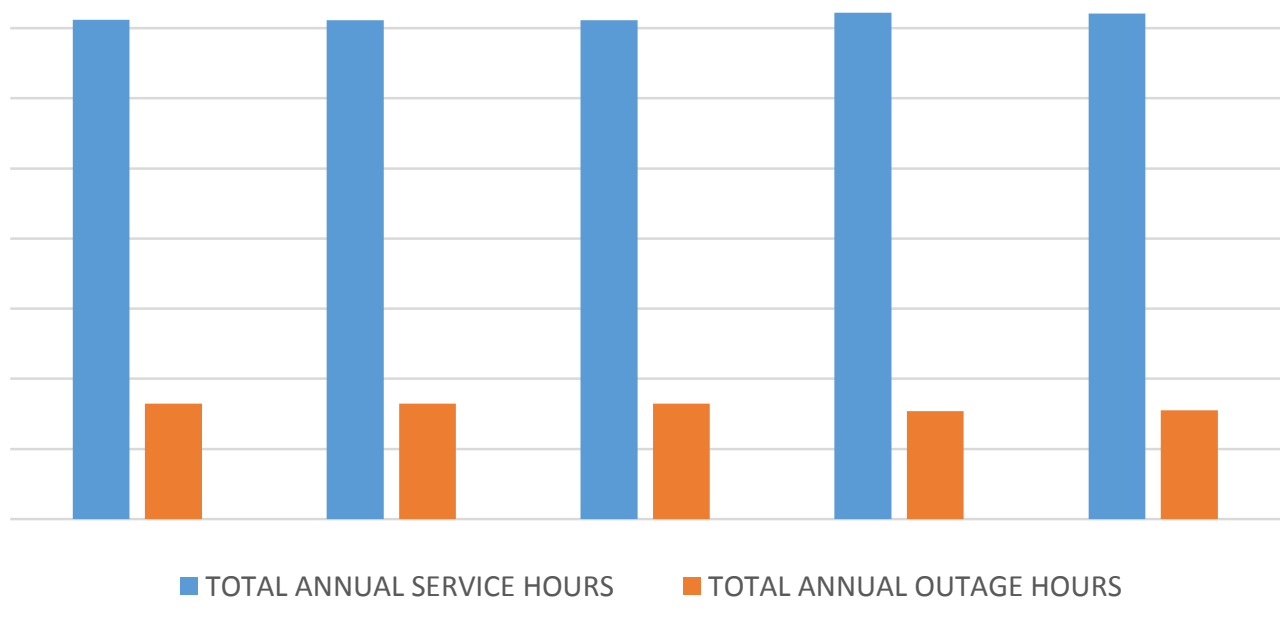

Fig. 3a: Service and Outage hours for the averagely performing feeders

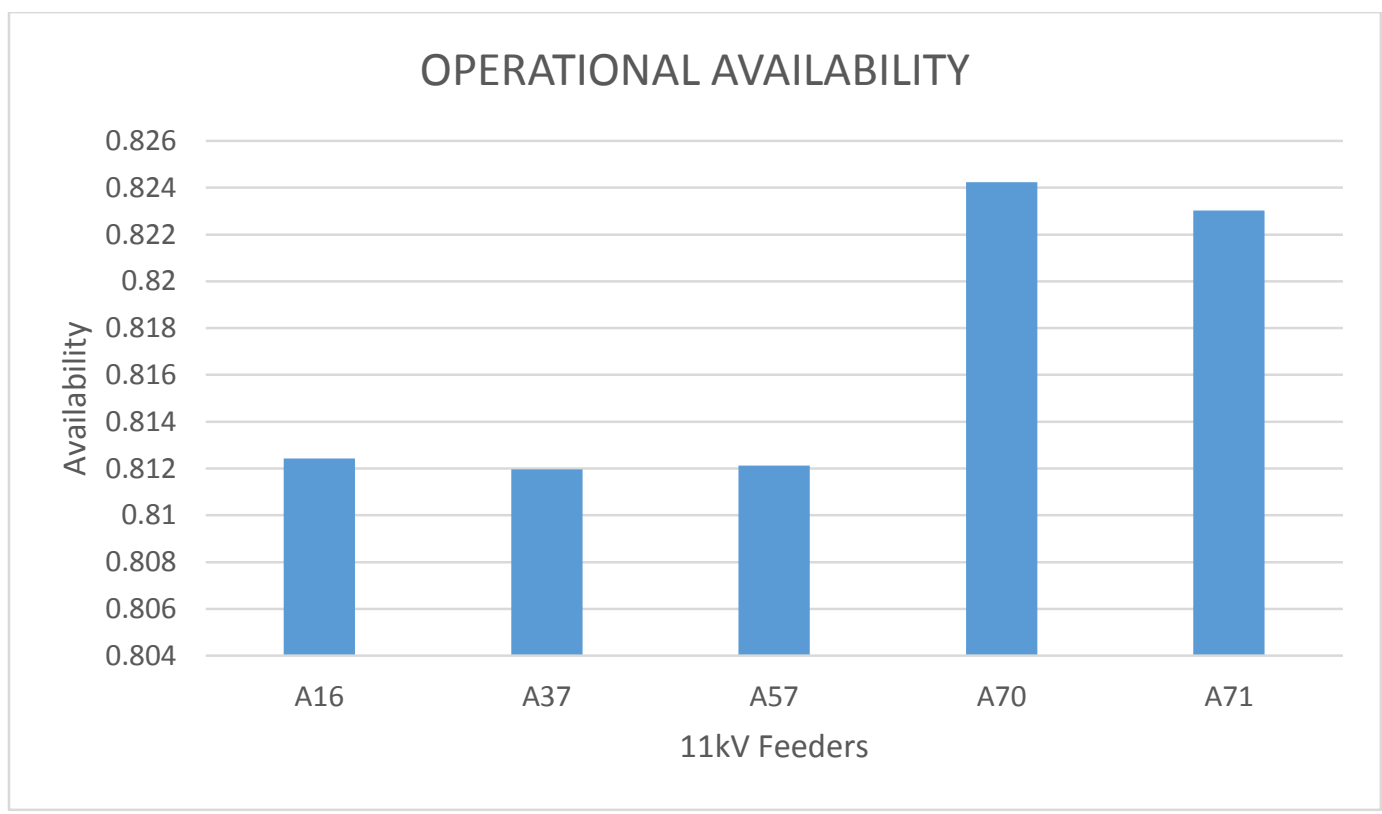

Fig.3b: Operational availability for the averagely performing feeders 
International Journal of Engineering Research and Technology. ISSN 0974-3154, Volume 13, Number 8 (2020), pp. 1940-1956 (C) International Research Publication House. https://dx.doi.org/10.37624/IJERT/13.8.2020.1940-1956

Table 8: Operational availability for the poorly performing feeders

\begin{tabular}{|c|c|c|c|c|}
\hline $\begin{array}{c}\text { FEEDER } \\
\text { NAME }\end{array}$ & $\begin{array}{c}\text { ANNUAL NO } \\
\text { OF FAULTS }\end{array}$ & $\begin{array}{c}\text { TOTAL ANNUAL } \\
\text { SERVICE HOURS }\end{array}$ & $\begin{array}{c}\text { TOTAL ANNUAL } \\
\text { OUTAGE HOURS }\end{array}$ & $\begin{array}{c}\text { OPERATIONAL } \\
\text { AVAILABILITY }\end{array}$ \\
\hline A46 & 35 & 6389.491 & 2370.509 & 0.729394 \\
\hline A47 & 32 & 6382.855 & 2377.145 & 0.728636 \\
\hline A48 & 90 & 6374.891 & 2385.109 & 0.727727 \\
\hline A49 & 15 & 6319.145 & 2440.855 & 0.721364 \\
\hline A50 & 13 & 6304.545 & 2455.455 & 0.719697 \\
\hline
\end{tabular}

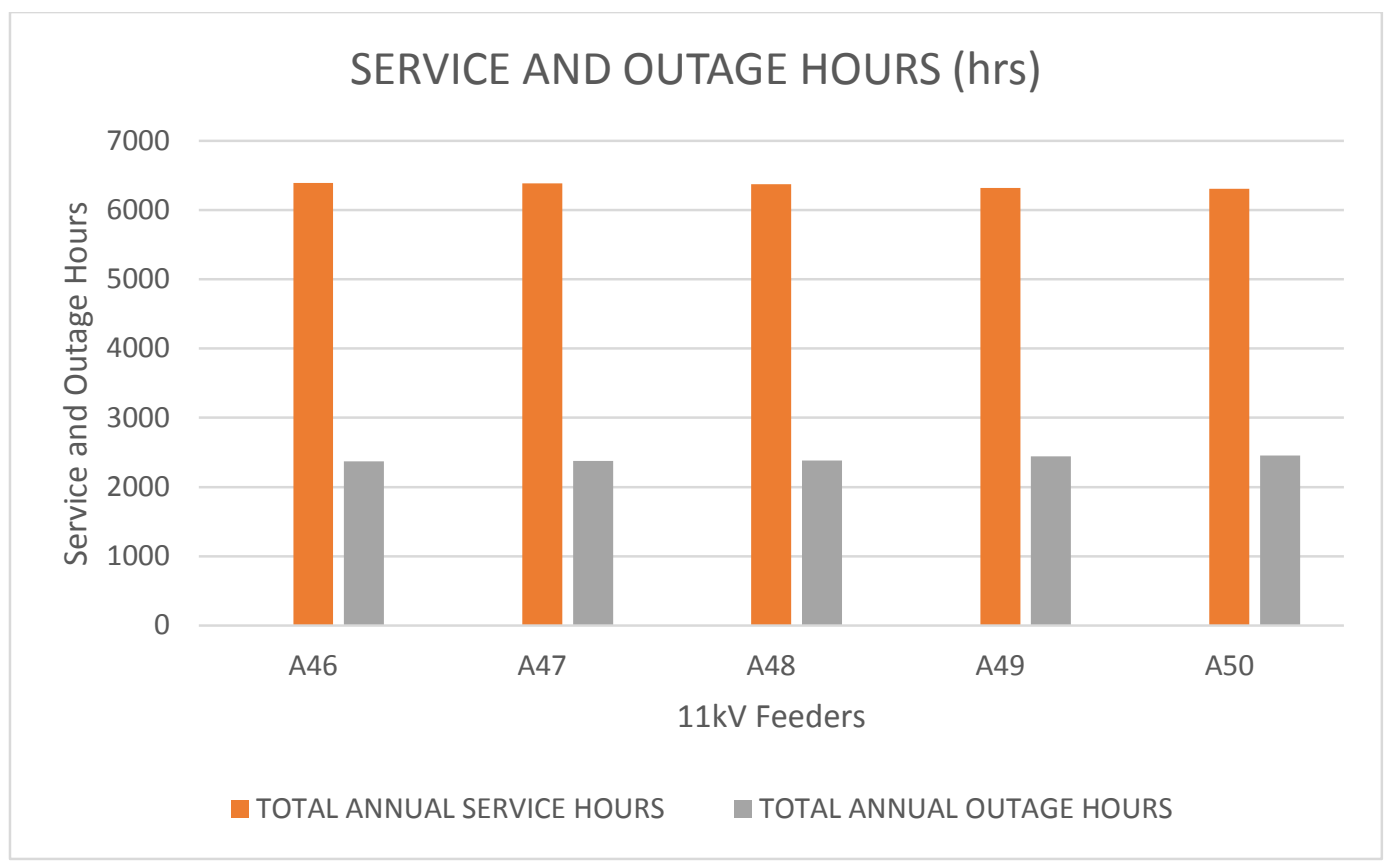

Fig. 4a: Service and Outage hours for the poorly performing feeders

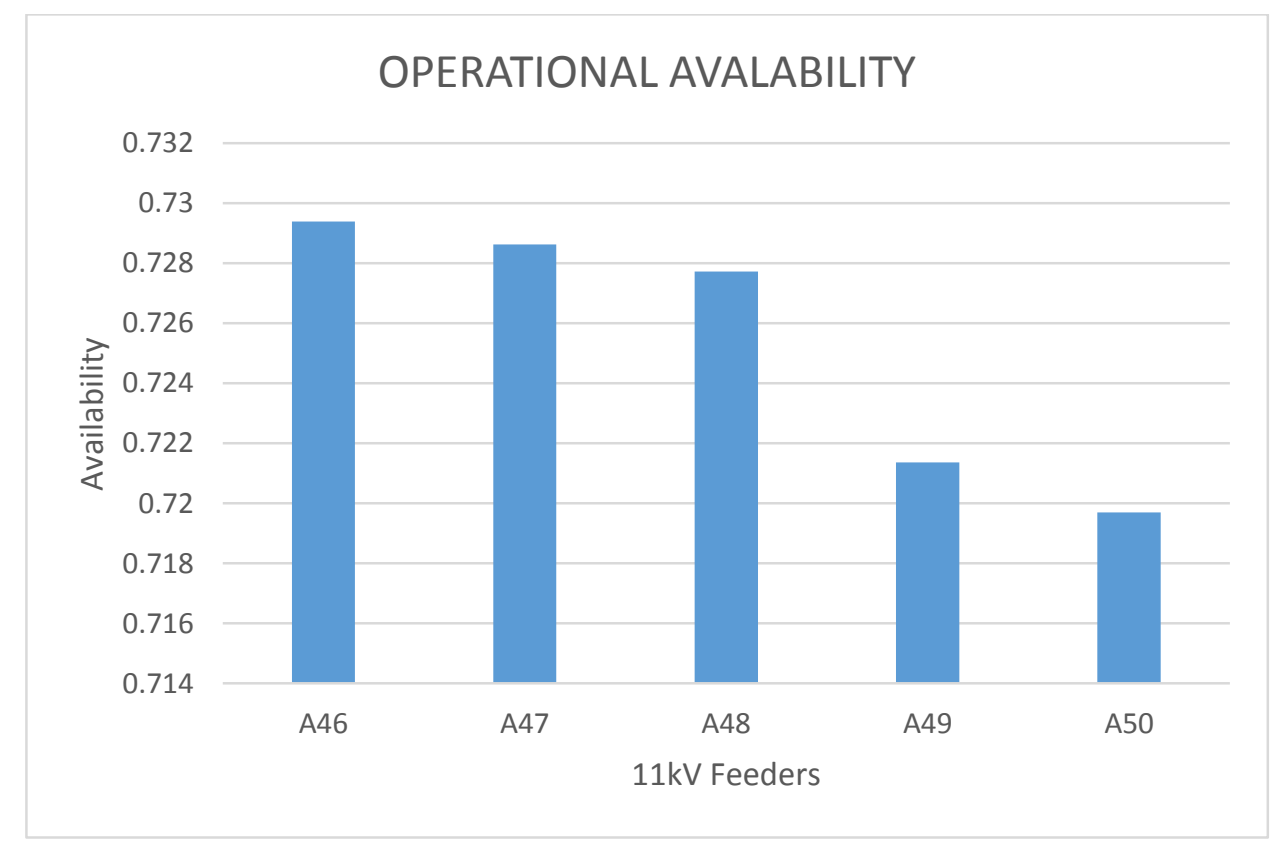

Fig. 4b: Operational availability for the poorly performing feeders 
International Journal of Engineering Research and Technology. ISSN 0974-3154, Volume 13, Number 8 (2020), pp. 1940-1956 (C) International Research Publication House. https://dx.doi.org/10.37624/IJERT/13.8.2020.1940-1956

Table 9: Feeders with best MTTF and MTBF values

\begin{tabular}{|c|c|c|c|c|c|}
\hline $\begin{array}{c}\text { FEEDER } \\
\text { NAME }\end{array}$ & MTTF (hrs) & $\begin{array}{c}\text { REPAIR RATE } \\
\text { (per hr) }\end{array}$ & $\begin{array}{c}\text { FAILURE } \\
\text { RATE (per hr) }\end{array}$ & MTTR (hrs) & MTBF (hrs) \\
\hline A1 & 8491.89091 & 0.15068 & 0.00012 & 6.63636 & 8498.52727 \\
\hline A3 & 8246.34545 & 0.07534 & 0.00012 & 13.27273 & 8259.61818 \\
\hline A2 & 4156.35455 & 0.04432 & 0.00024 & 22.56364 & 4178.91818 \\
\hline A5 & 2031.39091 & 0.05796 & 0.00049 & 17.25455 & 2048.64545 \\
\hline A37 & 1422.57 & 0.01 & 0.00070 & 98.33 & 1521.40 \\
\hline A70 & 1031.48 & 0.02 & 0.00097 & 65.96 & 1097.44 \\
\hline
\end{tabular}

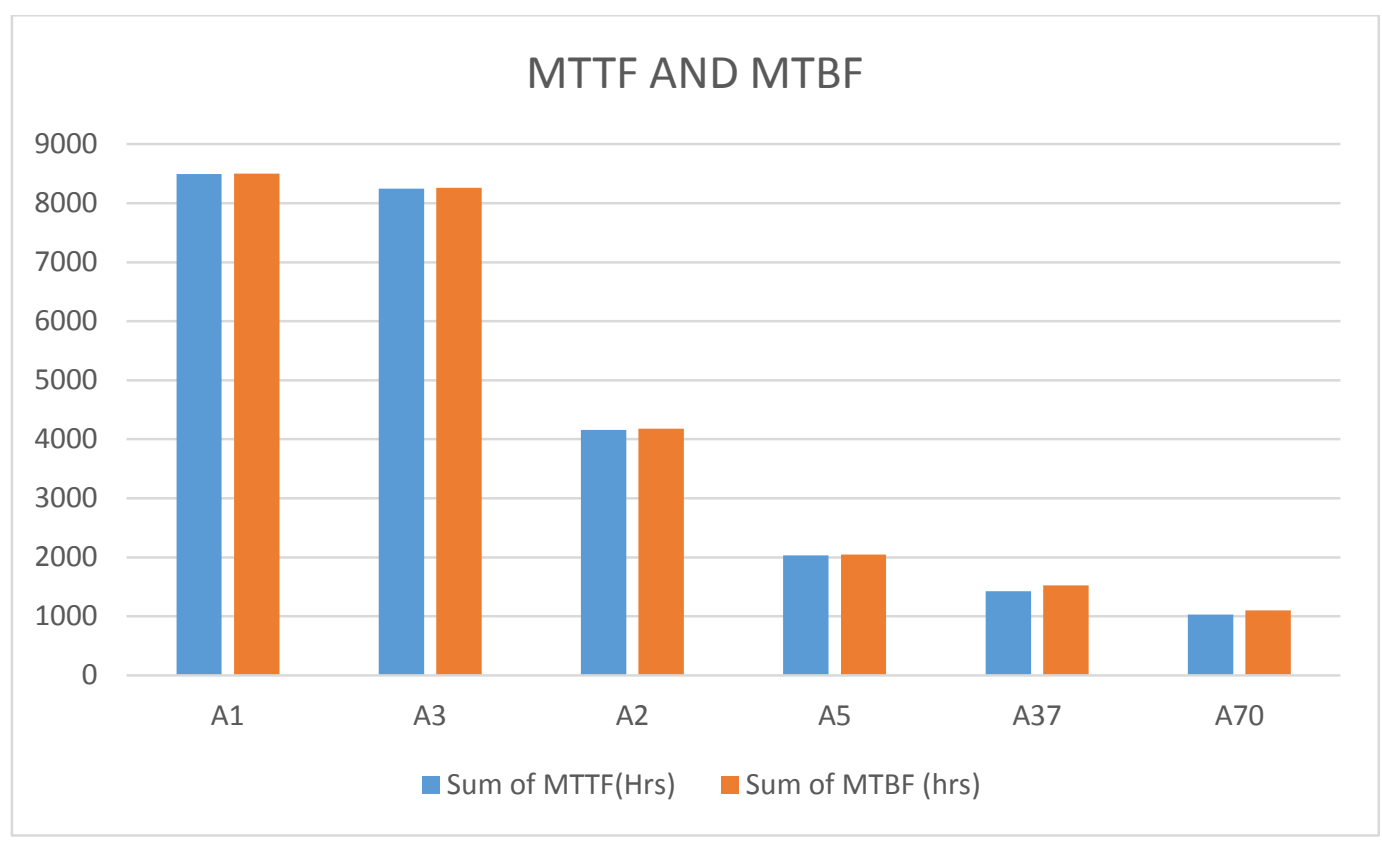

Fig.5a: Feeders with best MTTF and MTBF values

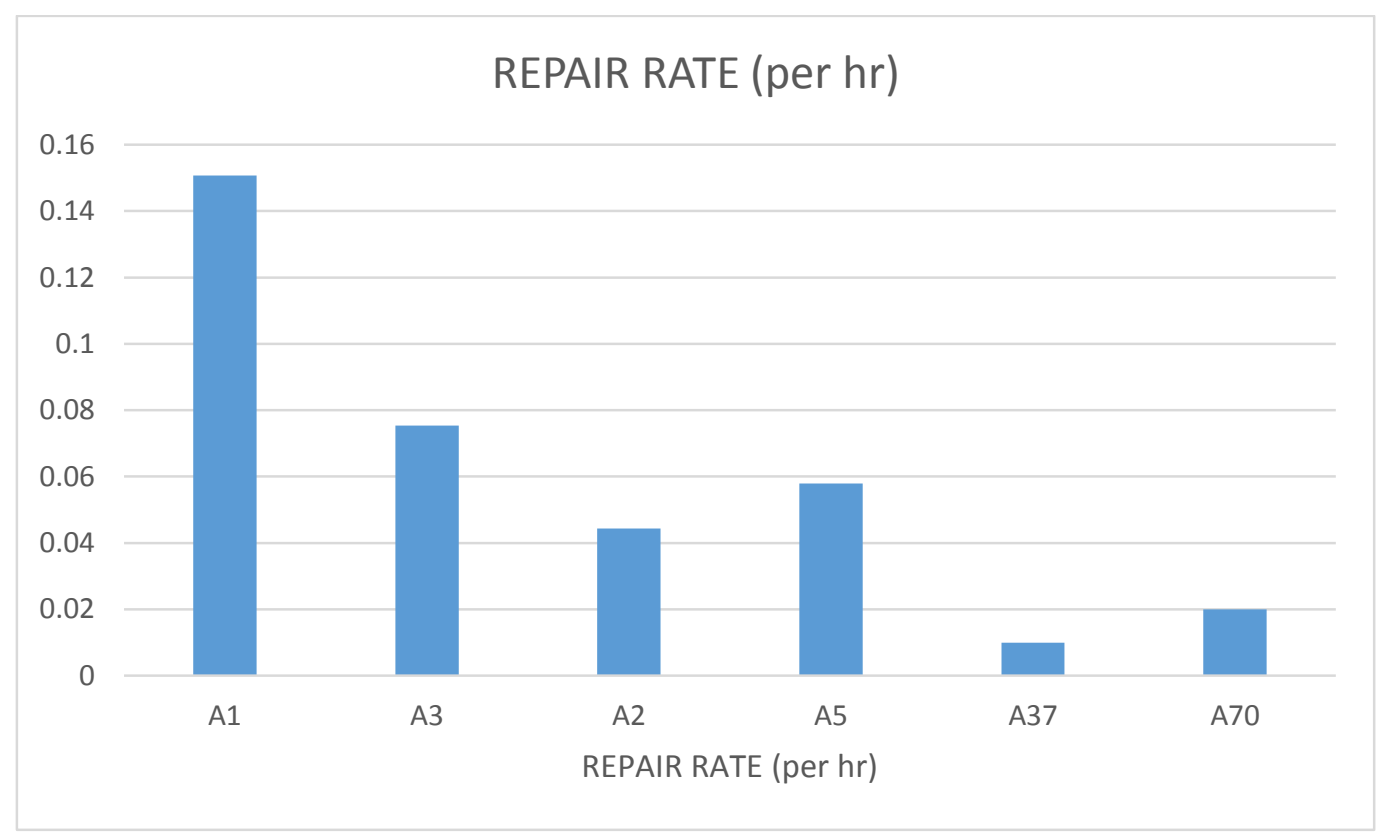

Fig. 5b: Feeders with best MTTF and MTBF values 
International Journal of Engineering Research and Technology. ISSN 0974-3154, Volume 13, Number 8 (2020), pp. 1940-1956 (C) International Research Publication House. https://dx.doi.org/10.37624/IJERT/13.8.2020.1940-1956

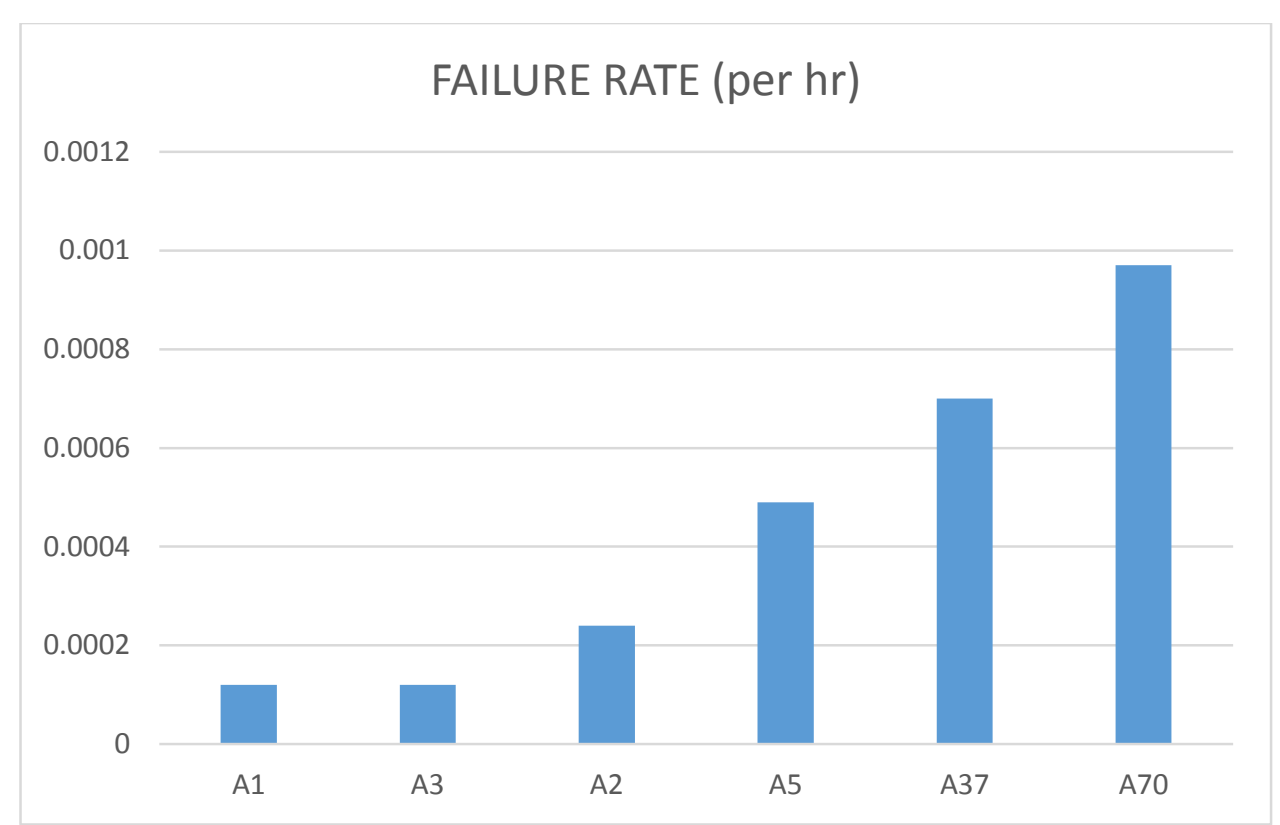

Fig. 5c: Feeders with best MTTF and MTBF values

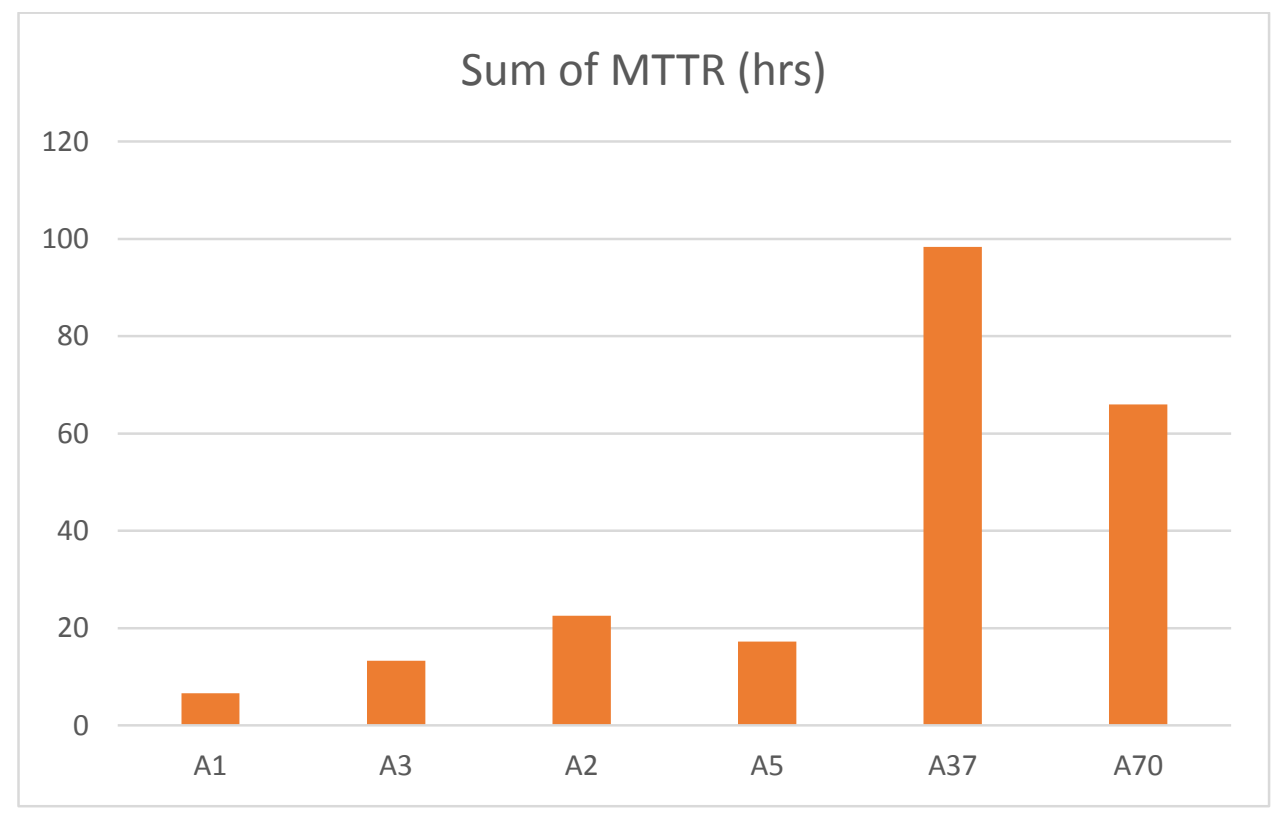

Fig. 5d: Feeders with best MTTF and MTBF values

Table 10: Feeders with averagely MTTF and MTBF

\begin{tabular}{|c|c|c|c|c|c|}
\hline $\begin{array}{c}\text { FEEDER } \\
\text { NAME }\end{array}$ & $\begin{array}{c}\text { MTTF } \\
\text { (hrs })\end{array}$ & $\begin{array}{c}\text { REPAIR RATE } \\
\text { (per hr) }\end{array}$ & $\begin{array}{c}\text { FAILURE RATE } \\
\text { (per hr) }\end{array}$ & $\begin{array}{c}\text { MTTR } \\
\text { (hrs })\end{array}$ & $\begin{array}{c}\text { MTBF } \\
\text { (hrs })\end{array}$ \\
\hline A4 & 746.65 & 0.07 & 0.00134 & 14.91 & 761.56 \\
\hline A49 & 421.27636 & 0.05486 & 0.00237 & 18.22788 & 439.50424 \\
\hline A50 & 484.97 & 0.02 & 0.00206 & 49.11 & 534.08 \\
\hline
\end{tabular}


International Journal of Engineering Research and Technology. ISSN 0974-3154, Volume 13, Number 8 (2020), pp. 1940-1956 (C) International Research Publication House. https://dx.doi.org/10.37624/IJERT/13.8.2020.1940-1956

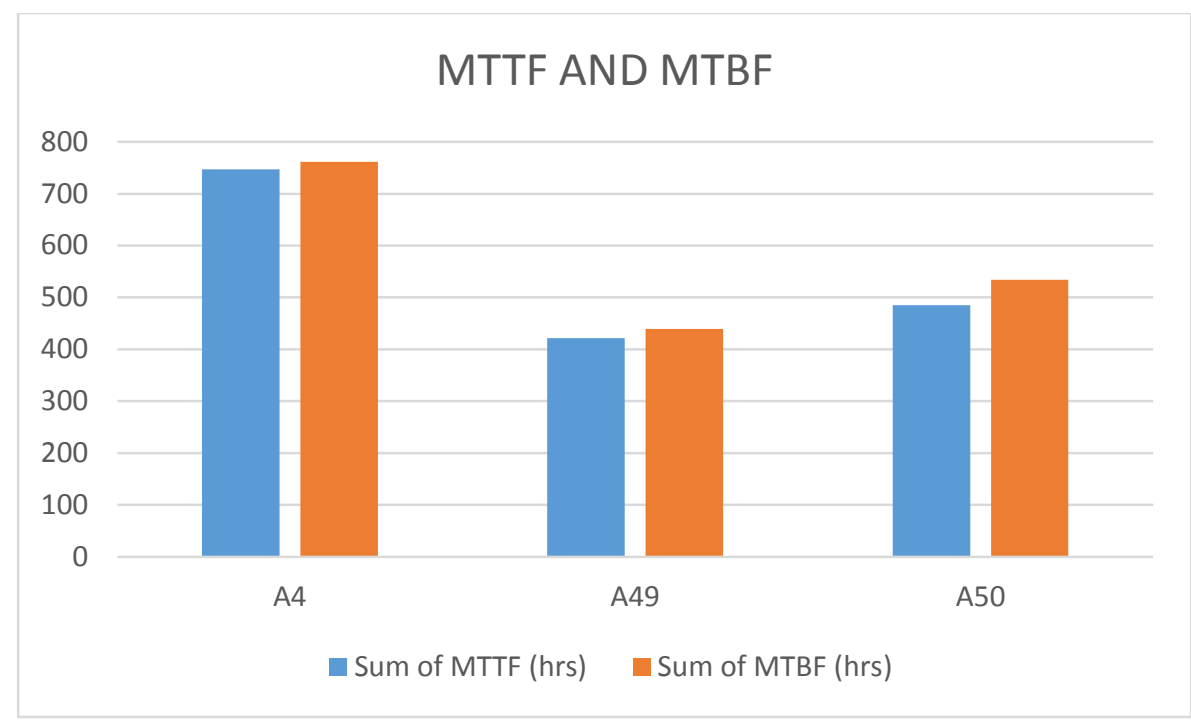

Fig. 6a: Feeders with average MTTF and MTBF

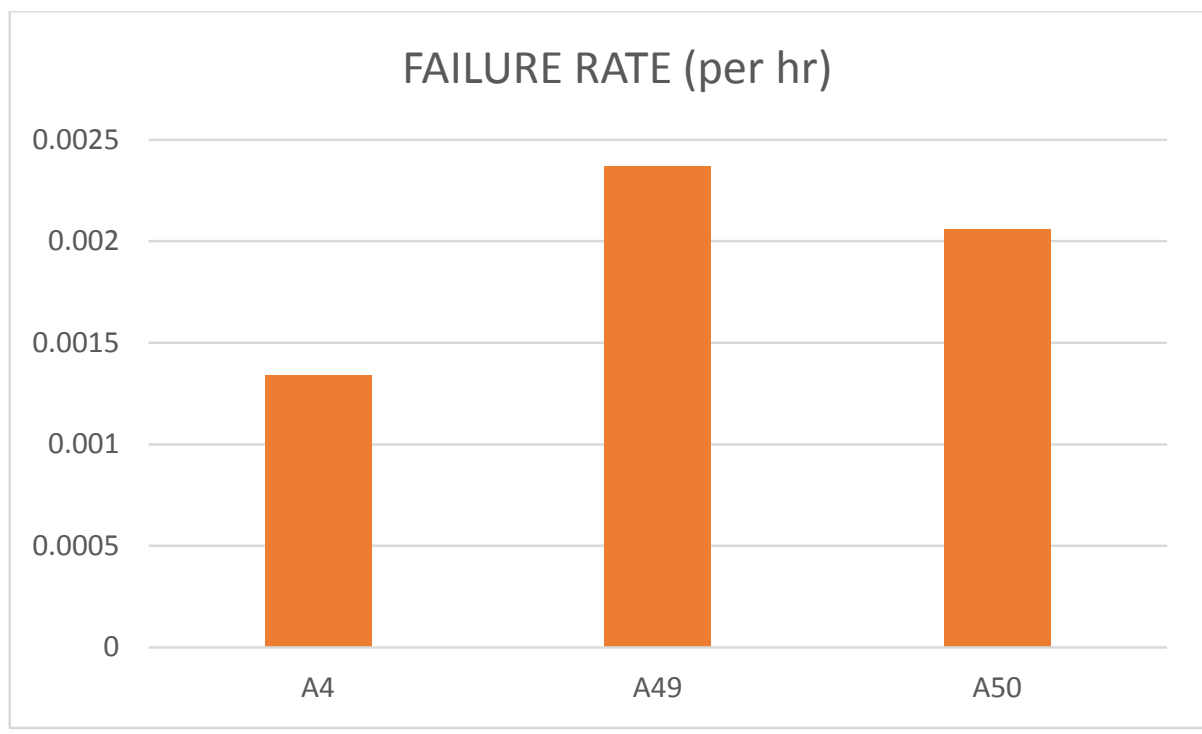

Fig. 6b: Feeders with average MTTF and MTBF

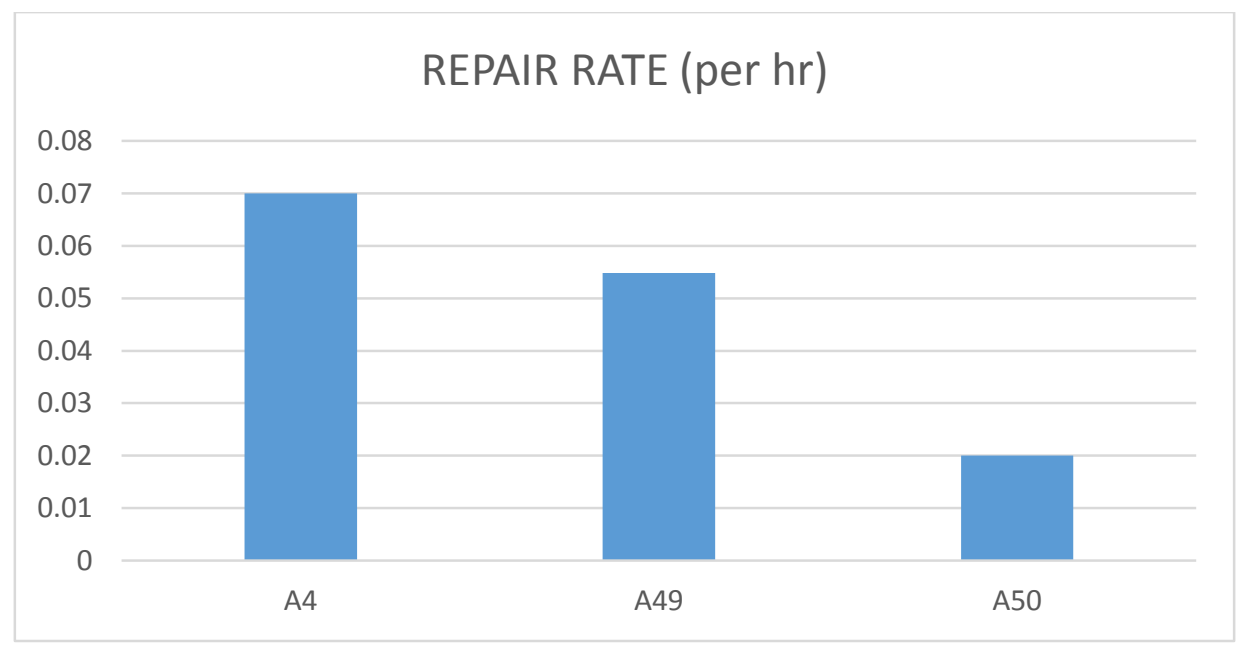

Fig. 6c: Feeders with average MTTF and MTBF 
International Journal of Engineering Research and Technology. ISSN 0974-3154, Volume 13, Number 8 (2020), pp. 1940-1956 (C) International Research Publication House. https://dx.doi.org/10.37624/IJERT/13.8.2020.1940-1956

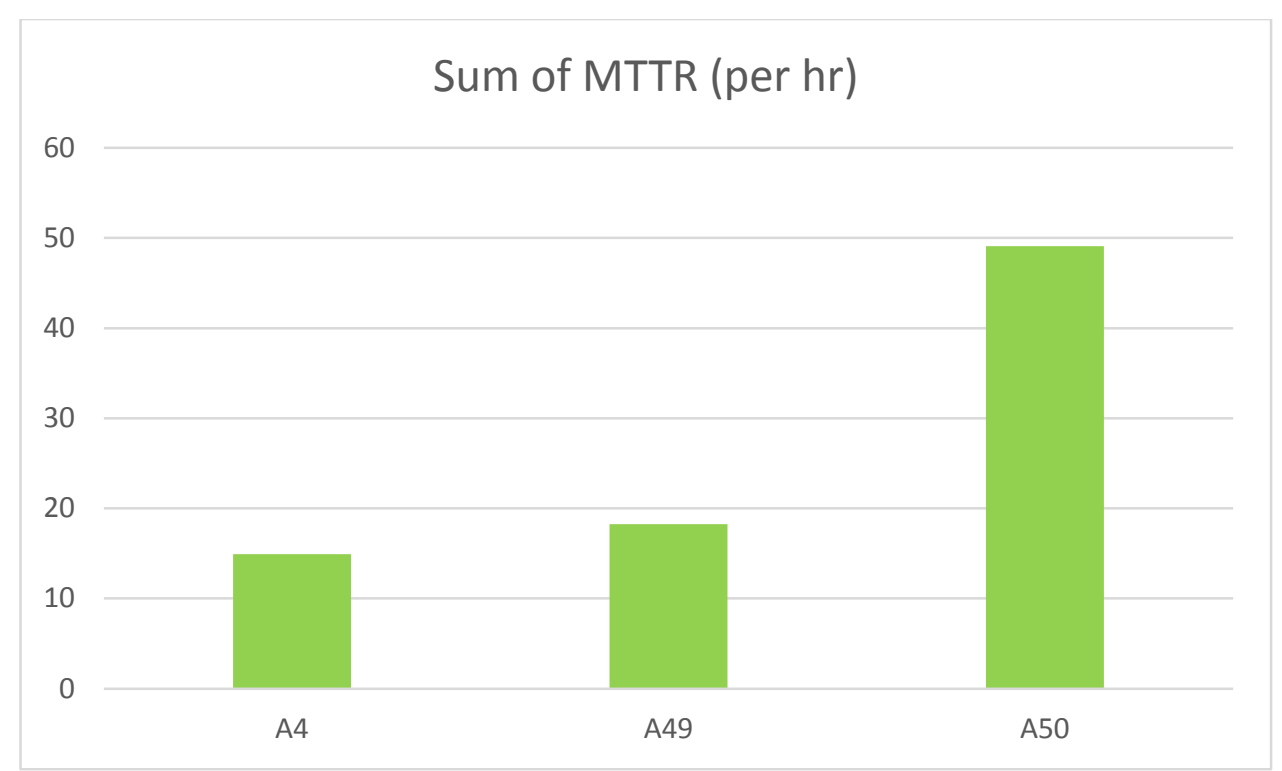

Fig. 6d: Feeders with average MTTF and MTBF

Table 11: Feeders with poor MTTF and MTBF values

\begin{tabular}{|c|c|c|c|c|c|}
\hline $\begin{array}{c}\text { FEEDER } \\
\text { NAME }\end{array}$ & $\begin{array}{c}\text { MTTF } \\
\text { (hrs) }\end{array}$ & $\begin{array}{c}\text { REPAIR RATE } \\
\text { (per hr) }\end{array}$ & $\begin{array}{c}\text { FAILURE RATE } \\
\text { (per hr) }\end{array}$ & $\begin{array}{c}\text { MTTR } \\
\text { (hrs })\end{array}$ & $\begin{array}{c}\text { MTBF } \\
(\mathrm{hrs})\end{array}$ \\
\hline A16 & 273.72 & 0.05 & 0.00365 & 18.96 & 292.68 \\
\hline A46 & 182.56 & 0.050 & 0.00548 & 20.32 & 202.88 \\
\hline A47 & 199.46 & 0.04 & 0.00501 & 22.29 & 221.75 \\
\hline A48 & 70.83 & 0.13 & 0.01 & 7.75 & 78.78 \\
\hline A57 & 197.62 & 0.07 & 0.00506 & 13.72 & 211.54 \\
\hline A37 & 1422.57 & 0.01 & 0.00070 & 98.33 & 1521.40 \\
\hline
\end{tabular}

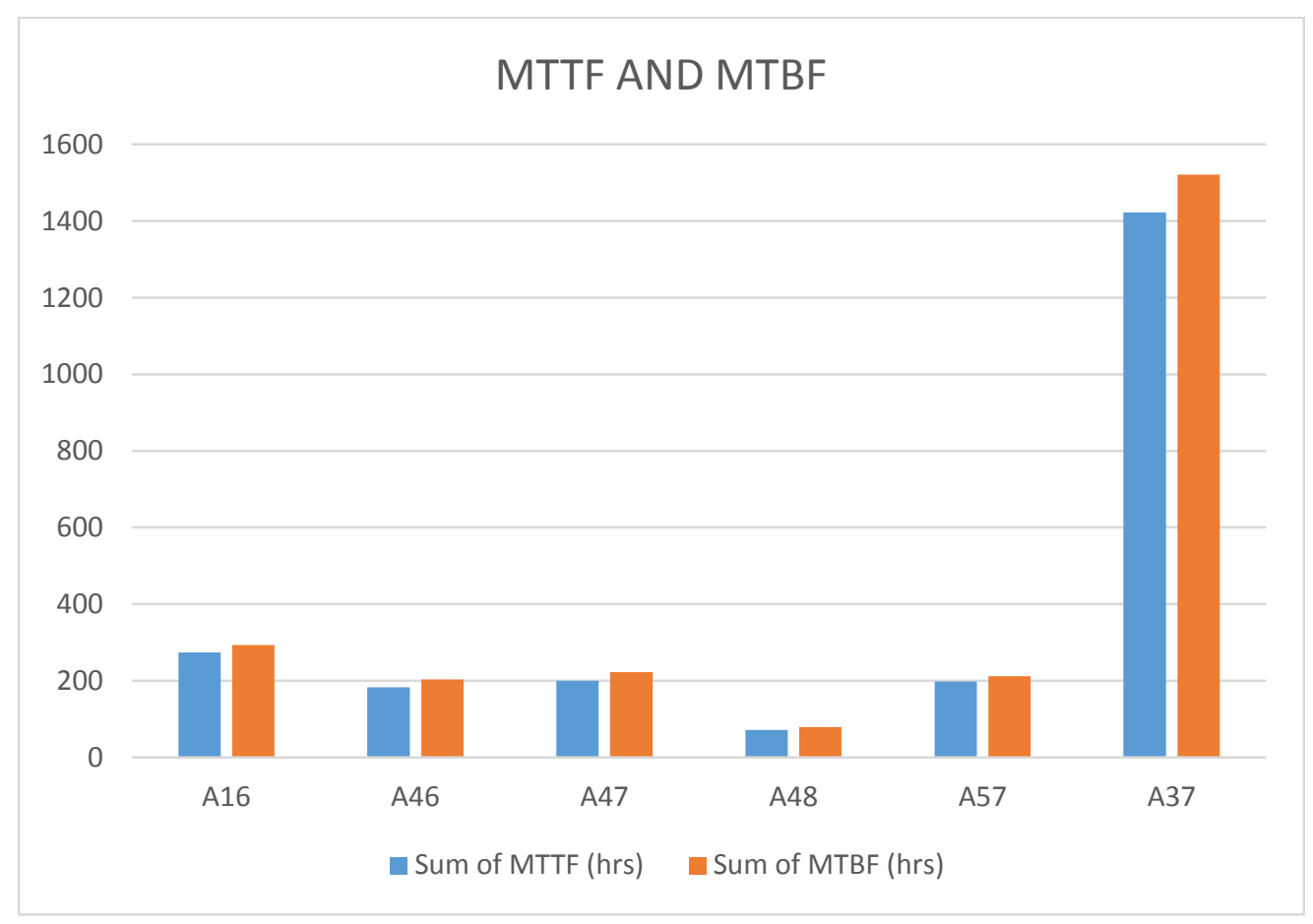

Fig. 7a: Feeders with poor MTTF and MTBF values 
International Journal of Engineering Research and Technology. ISSN 0974-3154, Volume 13, Number 8 (2020), pp. 1940-1956 (C) International Research Publication House. https://dx.doi.org/10.37624/IJERT/13.8.2020.1940-1956

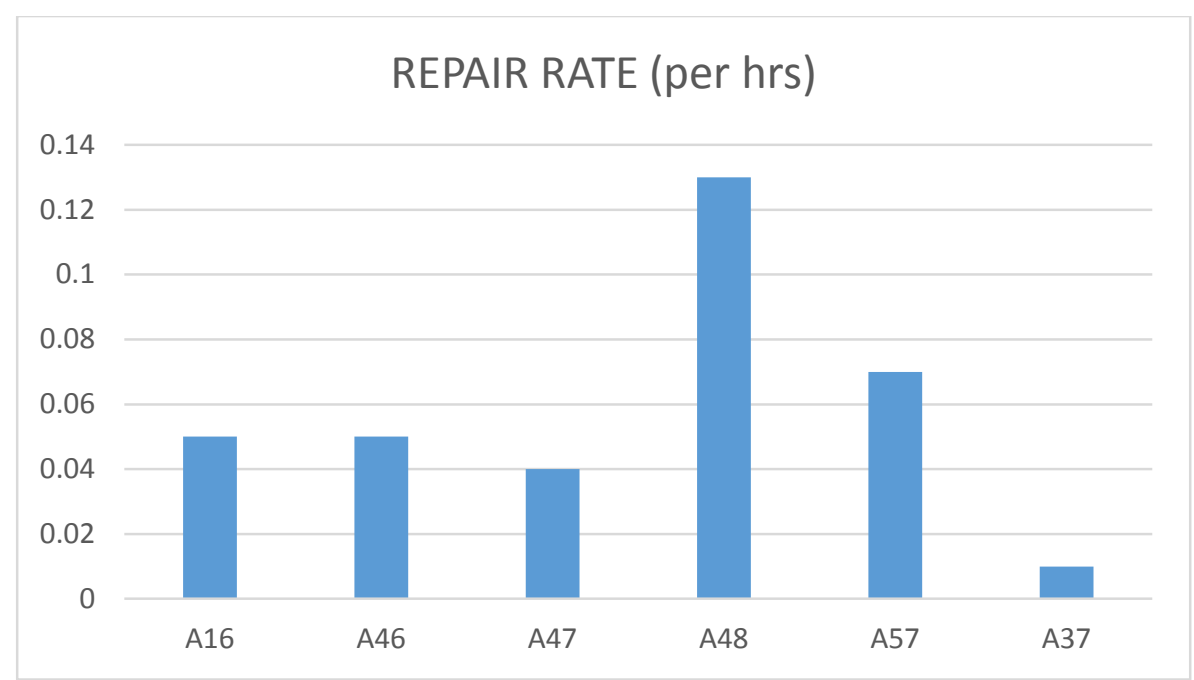

Fig. 7b: Feeders with poor MTTF and MTBF values

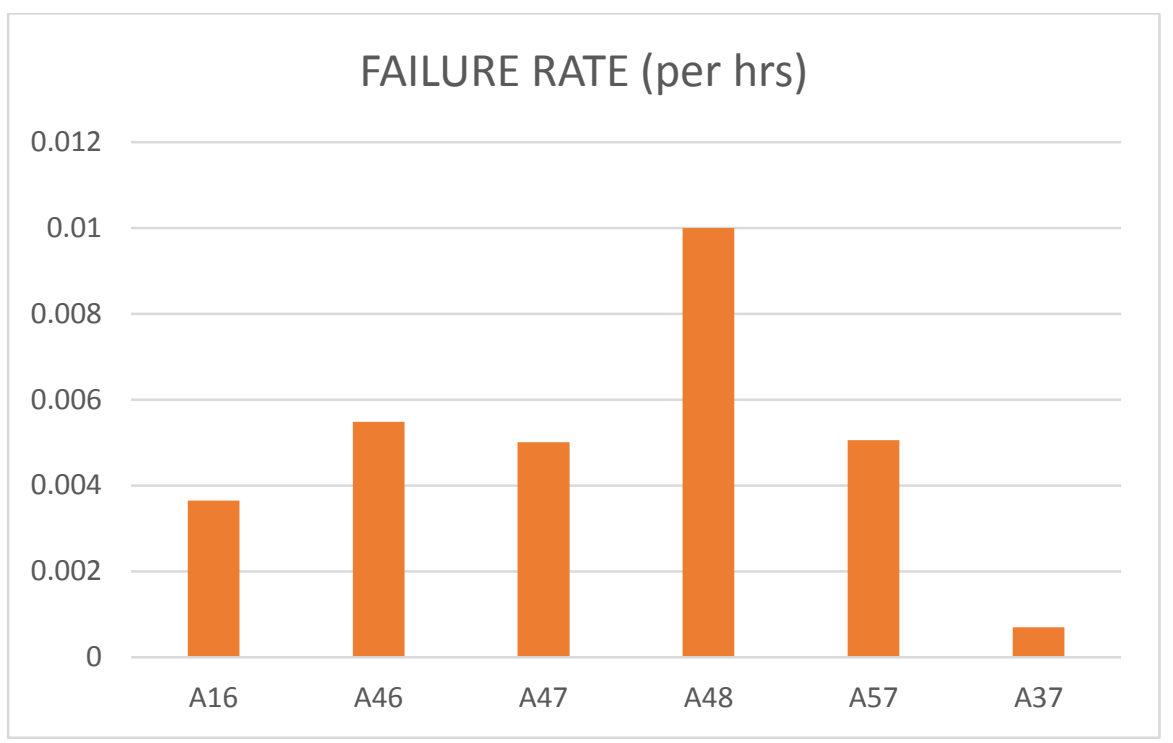

Fig. 7c: Feeders with poor MTTF and MTBF values

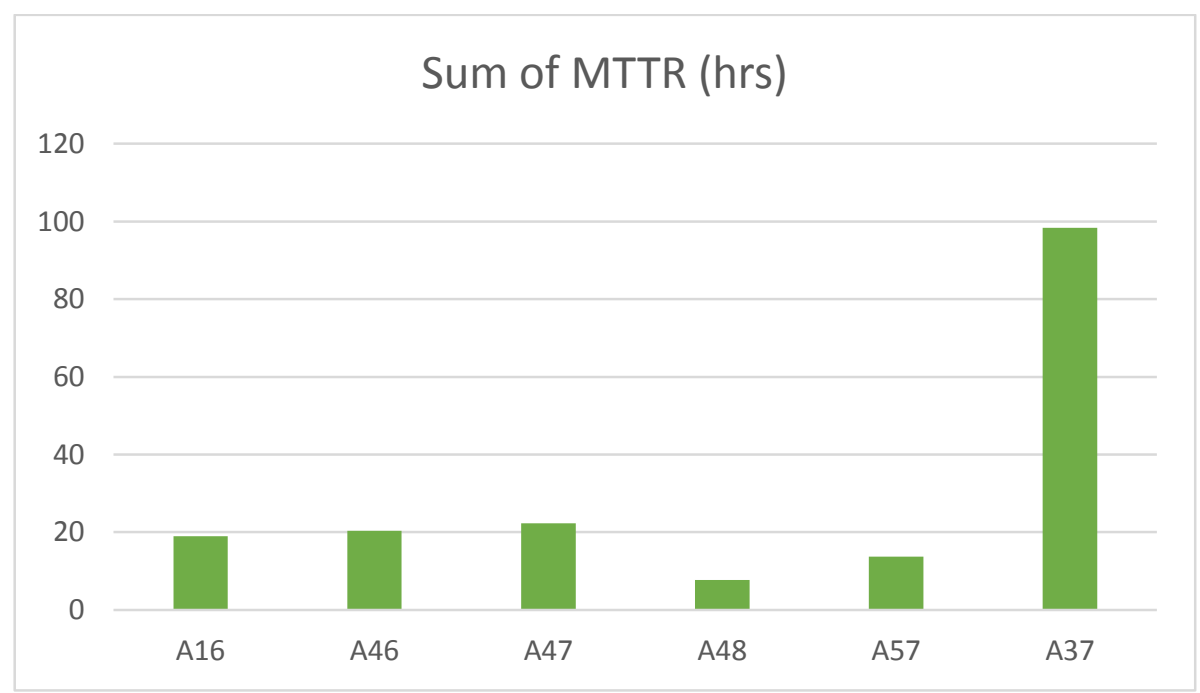

Fig. 7d: Feeders with poor MTTF and MTBF values 
International Journal of Engineering Research and Technology. ISSN 0974-3154, Volume 13, Number 8 (2020), pp. 1940-1956

(C) International Research Publication House. https://dx.doi.org/10.37624/IJERT/13.8.2020.1940-1956

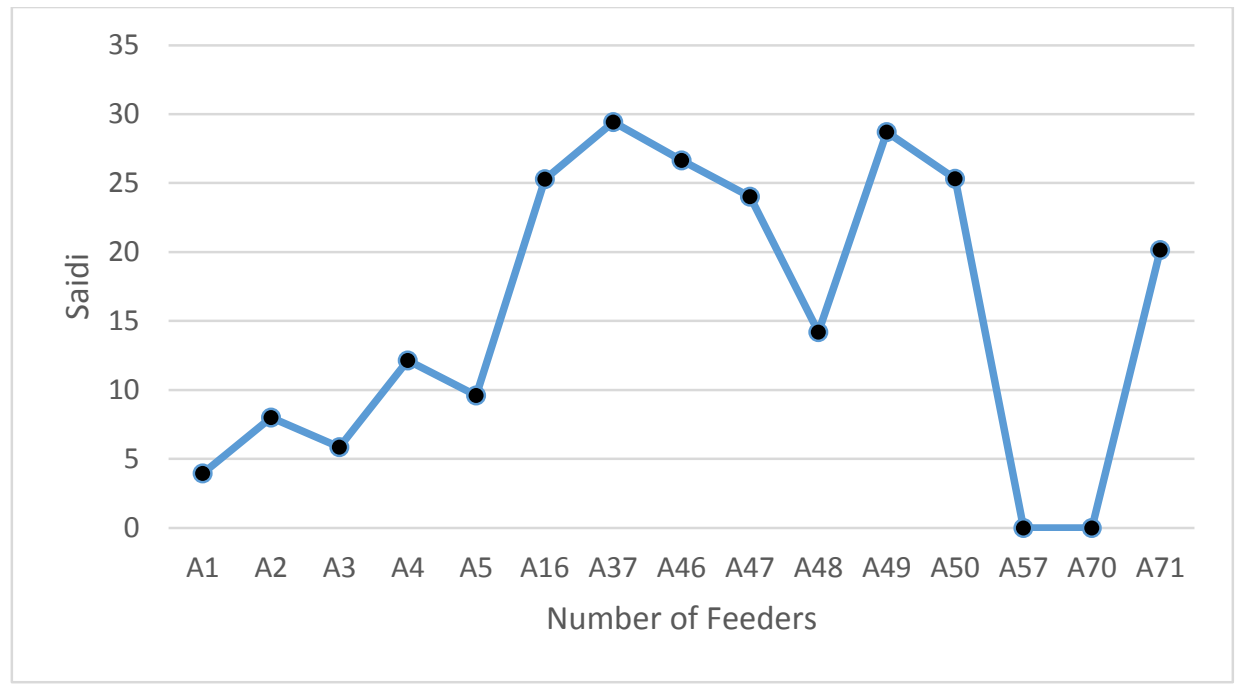

Fig. 8: Contribution of SAIDI for the 15 No Selected $11 \mathrm{kV}$ Feeders

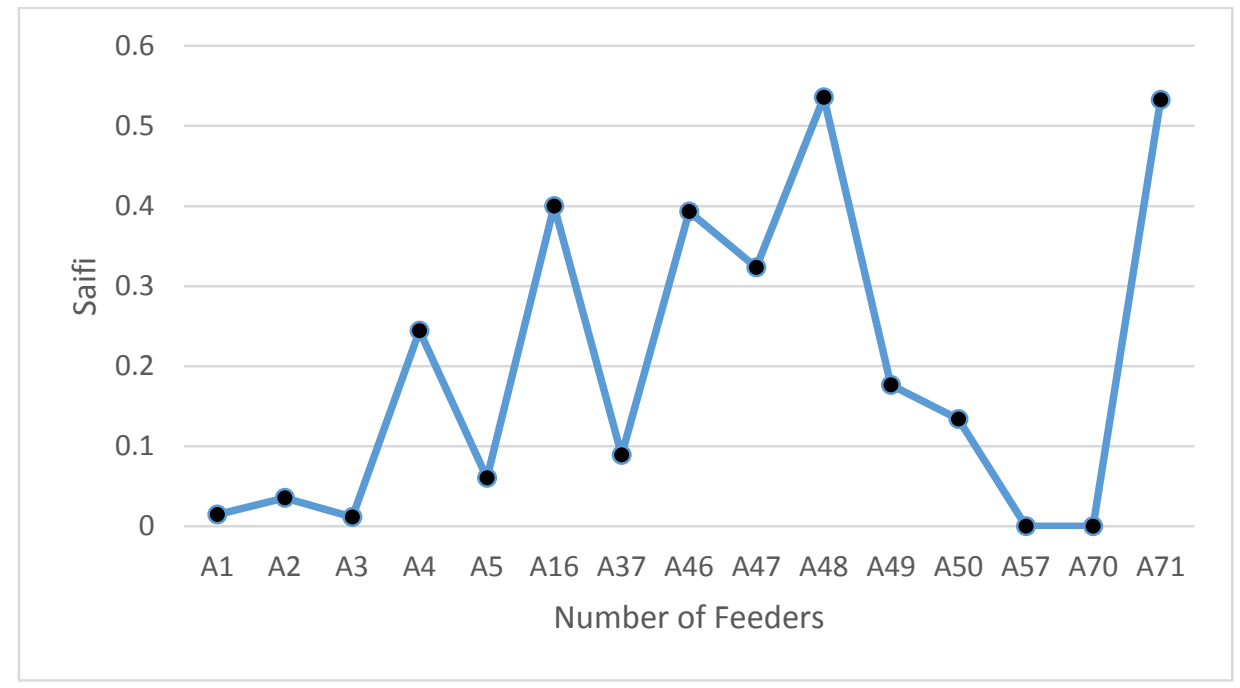

Fig. 9: Contribution of SAIFI for the 15 No. Selected $11 \mathrm{kV}$ Feeders

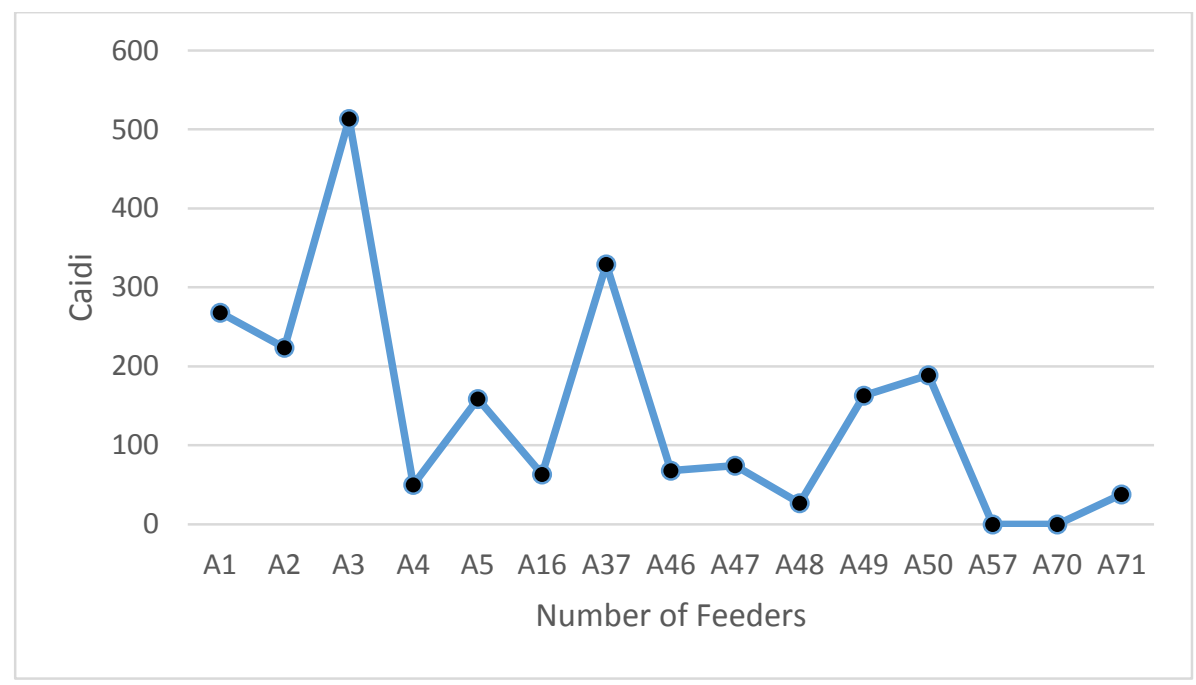

Fig. 10: Contribution of CAIDI for the 15No. Selected 11kV Feeders 


\section{RESULTS AND DISCUSSION}

Table 4 and 5 shows the reliability indices evaluated in the assessment of the case studied networks. Looking through table 4 , the performance of the feeders was categorised into three classes based on the results obtained as operational availability value for each of the feeders. These classes are the best performing feeders (group 1), the averagely performing feeders (Group 2) and the poorly performing feeders (Group 3). Feeders A1 to A5 are in group 1, Feeders A16, A37, A57, A70 and A71 are in group 2 while feeder A46 to A50 falls in group 3. The operational availability is probability that a system or equipment when used under stated conditions in an actual operational environment, shall operate satisfactorily at a given point in time [19, 24]. This parameter is the one that the customers normally experience during their dealings with power utility company because it is used to measure the efficiency of the service provider.

In Table 5, it is obvious that feeders in group 1, group 2 and group 3 have a considerable decreasing values of inherent availability of feeders' performance looking from group 1 to 3 . However, inherent availability is the probability that a system or equipment, when used under stated conditions, without consideration for any scheduled maintenance or preventive action, in an ideal support environment (i.e available tools. Spares, personnel, data. Etc.) Shall operate satisfactorily at a point in time [19, 24]. Therefore, it is a product of design and considers only corrective maintenance downtime. This imply, it excludes ready time, preventive maintenance downtime, logistics time and administrative downtime. The ready time is the time during which the system is in a functionally acceptable condition but is inactive or off.

Tables 6 to 8 displays extract of the best, averagely and poorly performing feeders in the area of concentration for this research work based on their service hours, outage hours, and electricity supply operational availability. From each of these tables, bar charts were plotted to give a graphical illustration of the results. The group 1 feeders have total service hours that are above 8000 hours, which is more than $90 \%$. The feeder with the best service hour is A1. These five feeders generally have low outage hours, which is a good index. The group 2 feeders have ensuing charts which suggest that the averagely performing feeders have good service hour values and almost similar outage hours. The group 3 which contains three poorly performing feeders. A50 feeder has the poorest values for total service hours.

Table 9 to 11 also displays an extract of the best, averagely and poorly performing feeders in the area of MTTF and MTBF. This time around the purported group classification did not work these indices. Feeders A1, A2, A3, A5, A37 and A70 gives the best MTTF and MTBF, feeders A4, A49 and A50 gives the averagely good MTTF and MBTF, while feeders with poor MTTF and MBTF are A16, A37, A46, A47, A48 and A57. Also from each of these tables, bar charts were plotted to give a graphical illustration of the results in the same way as above. Furthermore, low values for mean time to failure (MTTF) indicate that the time taken for the feeders to trip on fault again after restoration is extremely small and suggests that there are weak joints and poor maintenance on such feeders. Such feeders must be worked on. A large mean time to repair (MTTR) value denotes that the faults that happen on such feeders are such that take much time before they can be cleared. Based on this, A1 feeder has the lowest MTTR value of 6.63636, indicating that it does not take too long for faults to be cleared. Conversely, A37 feeder has the largest MTTR value of 98.33, suggesting that it takes quite long before faults on this feeder are cleared. The contribution of SAIDI, SAIFI and CAIDI to the fifteen $11 \mathrm{kV}$ feeders' network are graphically illustrated in Figures 8, 9 and 10 respectively.

From the discussion of results above, several feeders have been identified as those which needs their reliabilities to be improved. The following ways has however been suggested to improve the reliability of the affected feeders:

1. Prevent failures from occurring (by Feeder Reinforcement, creating optimum number of Opening Points/Sections and Installing Automatic/Manual Switches or Fuses/Cutouts, and Fusing all Taps-Off the Main Line etc.)

2. Reduce the number of affected customers (by Sectionalizing and Interconnecting with adjacent feeders etc.)

3. After fault occurs, restore more customers ( by Automation or Prompt Switching in case of Manual Operations as soon as the faulty components or Sections are identified and isolated)

4. After fault occurs, restore more quickly (by Automation i.e. use of Reclosers/Sectionalizers or Prompt Manual Switching)

5. Locate fault more quickly ( by Automation of Fault location process or Prompt response by the Protection \&Testing Crew)

6. Repair fault more quickly. (Prompt response of the Maintenance Crew or Field Engineers)

\section{CONCLUSION}

The paper has presented an evaluation of reliability parameters of a selected distribution network. This attempt ultimately ensure a robust distribution network of the case studied network considered. A Nigerian power distribution company having $15 \mathrm{No} 11 \mathrm{kV}$ feeders has been extensively analyzed as case studied. The results show that for a feeder to have high reliability index, the outage hours must be very low, very good service hours, low MTTF and availability of supply. To further improve the network reliability indices, some suggestions that could assist the utility company which was used as case study have been presented. As the reliability indices i.e MTTR, MTTF, MTBF, etc. have been employed to identify the most critical feeders. Improvement done on these feeders will have the greatest impact on total system reliability.

\section{ACKNOWLEDGEMENTS}

We appreciate the contribution of Eko Electricity Distribution Company (EKEDC) through which the data used for this research work were obtained. 
International Journal of Engineering Research and Technology. ISSN 0974-3154, Volume 13, Number 8 (2020), pp. 1940-1956

(C) International Research Publication House. https://dx.doi.org/10.37624/IJERT/13.8.2020.1940-1956

\section{REFERENCES}

[1] J. J. Popoola, A. A. Ponnle and T. O. Ale, Reliability worth Assessment of Electric Power Utility in Nigeria: Residential Customer Survey Results, American Union Journal of Technology, Vol. 14, Issue3, 2011, pp. 217 224.

[2] P. U. Okorie and A. I. Abdu, Reliability Evaluation of Power Distribution Network System in Kano Metropolis of Nigeria, International Journal of Electrical and Electronic Science, Vol. 2, No. 1, 2015, pp. 1-5.

[3] A. E. Airoboman, E. A.Ogujor and I. Kokakwu, IEEE PES - IAS Power Africa, 2017, pp.99-104.

[4] F. Onime and G. Adegboyega, Reliability Analysis of Power Distribution System in Nigeria: A Case Study of Ekpoma Network, Edo State, International Journal of Electronics and Electrical Engineering, Vol. 2, No. 3, 2014, pp.175-182.

[5] U. Eminoglu and R. Uyan, Reliability Analyses of Electrical Distribution System: A Case Study, International Refereed Journal of Engineering and Science (IRJES), Vol. 5, Issue 12, 2016, pp.94-102.

[6] T. M. Aljohani and M. J. Beshir, Distribution System Reliability Analysis for Smart Grid Applications, Smart Grid and Renewable Energy, Vol. 8, 2017, pp.240-251

[7] A. A. Akintola and C. O. A. Awosope, Reliability Analysis of Secondary Distribution System in Nigeria: A Case Study of Ayetoro 1 Substation, Aguda, Lagos State, International Journal of Engineering and Science (IJES), Vol. 6, Issue 7, 2017, pp.13-21.

[8] S. Babu, Reliability Evaluation of Distribution Systems Considering Failure Modes and Network Configuration, Doctoral Thesis, Royal Institute of Technology, Stockholm, Sweden, 2017.

[9] H. Abunima, J. Teh, C. Lai and H. J. Jabir, A Systematic Review of Reliability Studies on Composite Power Systems: A Coherent Taxonomy Motivations, Open Challenges, Recommendations, and New Research Direction, MDPI Energie, 2018.

[10] T. O. Ale and A. O. Odesola, Unreliable Electric Power Supply in Nigeria (Akure $11 \mathrm{Kv}$ feeder a case study), International Journal of Emerging Engineering Research and Technology, Vol. 3, Issue 3, 2015, pp.68-75.

[11] N. Ali, U. D. Achinaya and M. S. Nuhu, Frequency failure of equipment of power system network- the Nigeria experience, Proc. $8^{\text {th }}$ IEE International Conference Dev. Power System Protection, 2(5-8), 2004, pp.603-609.

[12] T. O. Ale and A. O. Odesola, Effects of distance on transmission, Akure $33 \mathrm{kV}$ line a case study, International Journal of Research findings in Engineering Science and Technology, Vol. 1, No. 1, 2013, pp.27-32

[13] M. P. Bhavaraju, R. Billintom, R. E. Brown, J. Endrenyi, W. Li, A. P. Meliopoulous and C. Singh, IEEE
Tutorial on Electric delivery system reliability evaluation, Journal IEE Power Engineering Society (PES), publication, 305 TP 125, 2007, pp.39-51.

[14] D. N. Dewangan, M. J. Kumar and Y. P. Banjare, Reliability Investigation of Steam turbine used in Thermal Power Plant, International Journal of Innovative Research in Science, Engineering and Technology (IJIRSET), Vol. 3, 2014, pp.14915-14923.

[15] J. Barabady, Improvement of system availability using reliability and Maintainability analysis, Lulea University of Technology, Lulea, Sweden, 2005, pp.112.

[16] M. Z. Adamu, B. A. Adegboye, B. G. Bajoga, J. G. Ambafi and J. T. Omokhafe, Reliability Evaluation of Kainji hydro - electric power station in Nigeria, Journal of Energy Technologies and Policy, Vol. 2, 2012, pp.1530 .

[17] M. I. Abdulahi, O. Akinsanmi, M. B. Muazu and Y. Jubril, Reliability assessment of an electronic system: A case study of British Siren in Nigeria, Assumption University Journal of Thailand, Vol. 14, 2007, pp.217224.

[18] A. Kolawole, O. O. Agbolola, P. P. Ikubanni, O. G. Raji and C. O. Osueke, Reliability and power loss analysis: A case study of a power plant in Nigeria, Cogent Engineering Journal, Vol. 6, Issue 1, 2019.

http://www.tandfonline.com/doi/full/10.1080/23311916 .2019.157942.

[19] G. A. Ajenikoko, L. M. Adesina, O. S. Olaniyan and Y. A. Kosiru, Impact of Power distribution feeder's Contributions to Reliability Indices, Impact Journals: International Journal of Research in Engineering and Technology, ISSN(P): 2347 - 4599, ISSN(E): $2321-$ 8843, Vol. 7, Issue 11, 2019, pp.1-14.

[20] A. P. Meliopoulos, X. Chao, C. J. Georwe, R. Monsalvatge, Transmission loss evaluation based on probabilistic power flow, IEEE transactions on Power Systems, Vol. 6, No. 1, 2017, pp. 364-371.

G. Min, P. Anil and D. Sanjoy, Analysis of animalrelated outages in overhead distribution systems with wavelet decomposition and immune systems - based neural networks, IEEE transactions on power system, Vol. 24, No. 4, 2009, pp.1765-1770.

[22] Unpublished Outage Record Logbook of Operation and Maintenance Department, Eko Electricity Distribution Company (EKEDC), Marina - Lagos, Nigeria, 2019.

[23] Unpublished Test Record Logbook of Protection, Control and Metering (PC\&M) Department, Eko Electricity Distribution Company (EKEDC), Marina Lagos, Nigeria, 2019.

[24] Y. A. Adediran, Introduction to Reliability Engineering. Finom Associates, ISBN: 978 - 978 - 52341-2-1, First Edition, 2014 